\title{
Population study of Galactic supernova remnants at very high $\gamma$-ray energies with H.E.S.S.
}

H.E.S.S Collaboration: H. Abdalla ${ }^{1}$ A Abramowski ${ }^{2}$, F Aharonian ${ }^{3,4,5}$, F. Ait Benkhali ${ }^{3}$, E O Angüner ${ }^{21}$, M. Arakawa ${ }^{42}$, M. Arrieta ${ }^{15}$ P. Aubert ${ }^{24}$, M. Backes ${ }^{8}$, A. Balzer ${ }^{9}$, M. Barnard ${ }^{1}$, Y. Becherini ${ }^{10}$, J. Becker Tjus ${ }^{11}$, D. Berge ${ }^{12}$, S. Bernhard $^{13}$, K. Bernlöhr ${ }^{3}$, R. Blackwell ${ }^{14}$ M. Böttcher ${ }^{1}$, C. Boisson ${ }^{15}$, J. Bolmont ${ }^{16}$, S. Bonnefoy ${ }^{37}$, P. Bordas ${ }^{3}$, J. Bregeon ${ }^{17}$, F. Brun ${ }^{26}$, P. Brun ${ }^{18}$, M. Bryan ${ }^{9}$, M. Büchele ${ }^{36}$, T. Bulik ${ }^{19}$, M. Capasso ${ }^{29}$, S. Caroff ${ }^{30}$, A. Carosi ${ }^{24}$, S. Casanova ${ }^{21,3}$, M. Cerruti ${ }^{16}$, N. Chakraborty ${ }^{3}$, R. C. G. Chaves ${ }^{17,22, \star}$, A. Chen $^{23}$, J. Chevalier $^{24}$, S. Colafrancesco ${ }^{23}$, B. Condon ${ }^{26}$, J. Conrad ${ }^{27,28}$, I. D. Davids ${ }^{8}$, J. Decock ${ }^{18}$, C. Deil ${ }^{3}$, J. Devin ${ }^{17}$, P. deWilt ${ }^{14}$, L. Dirson ${ }^{2}$, A. Djannati-Atai ${ }^{31}$,

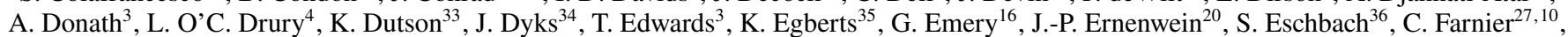
S. Fegan ${ }^{30}$, M. V. Fernandes ${ }^{2}$, D. Fernandez ${ }^{17}$, A. Fiasson ${ }^{24}$, G. Fontaine ${ }^{30}$, S. Funk ${ }^{36}$, M. Füßling ${ }^{37}$, S. Gabici ${ }^{31}$, Y. A. Gallant ${ }^{17}$, T. Garrigoux ${ }^{1}$, F. Gate ${ }^{24}$, G. Giavitto ${ }^{37}$, B. Giebels ${ }^{30}$, D. Glawion ${ }^{25}$, J. F. Glicenstein ${ }^{18}$, D. Gottschall ${ }^{29}$, M.-H. Grondin ${ }^{26}$, J. Hahn $^{3, \star}$, M. Haupt $^{37}$, J. Hawkes ${ }^{14}$,

G. Heinzelmann ${ }^{2}$, G. Henri ${ }^{32}$, G. Hermann ${ }^{3}$, J.A. Hinton ${ }^{3}$, W. Hofmann ${ }^{3}$, C. Hoischen ${ }^{35}$, T. L. Holch ${ }^{7}$, M. Holler ${ }^{13}$, D. Horns ${ }^{2}$, A. Ivascenko ${ }^{1}$,

H. Iwasaki ${ }^{42}$, A. Jacholkowska ${ }^{16}$, M. Jamrozy ${ }^{38}$, D. Jankowsky ${ }^{36}$, F. Jankowsky ${ }^{25}$, M. Jingo ${ }^{23}$, L. Jouvin ${ }^{31}$, I. Jung-Richardt ${ }^{36}$, M.A. Kastendieck ${ }^{2}$,

K. Katarzyński ${ }^{39}$, M. Katsuragawa ${ }^{43}$, U. Katz ${ }^{36}$, D. Kerszberg ${ }^{16}$, D. Khangulyan ${ }^{42}$, B. Khélifi ${ }^{31}$, J. King ${ }^{3}$, S. Klepser ${ }^{37}$, D. Klochkov ${ }^{29}$,

W. Kluźniak ${ }^{34}$, Nu. Komin ${ }^{23}$, K. Kosack ${ }^{18}$, S. Krakau ${ }^{11}$, M. Kraus ${ }^{36}$, P. P. Krüger ${ }^{1}$, H. Laffon ${ }^{26}$, G. Lamanna ${ }^{24}$, J. Lau ${ }^{14}$, J.-P. Lees ${ }^{24}$,

J. Lefaucheur ${ }^{15}$, A. Lemière ${ }^{31}$, M. Lemoine-Goumard ${ }^{26}$, J.-P. Lenain ${ }^{16},{\text { E. } \text { Leser }^{35} \text {, T. Lohse }}^{7}$, M. Lorentz ${ }^{18}$, R. Liu ${ }^{3}$, R. López-Coto ${ }^{3}$, I. Lypova ${ }^{37}$, D. Malyshev ${ }^{29}$, V. Marandon ${ }^{3, \star}$, A. Marcowith ${ }^{17}$, C. Mariaud ${ }^{30}$, R. Marx ${ }^{3}$, G. Maurin ${ }^{24}$, N. Maxted ${ }^{14,45}$, M. Mayer ${ }^{7}$, P. J. Meintjes ${ }^{40}$,

M. Meyer ${ }^{27,47}$, A. M. W. Mitchell ${ }^{3}$, R. Moderski ${ }^{34}$, M. Mohamed $^{25}$, L. Mohrmann ${ }^{36}$, K. Moră $^{27}$, E. Moulin ${ }^{18}$, T. Murach ${ }^{37}$, S. Nakashima $^{43}$,

M. de Naurois ${ }^{30}$, H. Ndiyavala ${ }^{1}$, F. Niederwanger ${ }^{13}$, J. Niemiec $^{21}$, L. Oakes ${ }^{7}$, P. O’Brien ${ }^{33}$, H. Odaka ${ }^{43}$, S. Ohm ${ }^{37}$, M. Ostrowski $^{38}$, I. Oya $^{37}$,

M. Padovani ${ }^{17}$, M. Panter ${ }^{3}$, R. D. Parsons ${ }^{3}$, N. W. Pekeur ${ }^{1}$, G. Pelletier ${ }^{32}$, C. Perennes ${ }^{16}$, P.-O. Petruccis ${ }^{32}$, B. Peyaud ${ }^{18}$, Q. Piel ${ }^{24}$, S. Pita ${ }^{31}$,

V. Poireau ${ }^{24}$, H. Poon ${ }^{3}$, D. Prokhorov ${ }^{10}$, H. Prokoph ${ }^{12}$, G. Pühlhofer ${ }^{29}$, M. Punch $^{31,10}$, A. Quirrenbach ${ }^{25}$, S. Raab $^{36}$, R. Rauth ${ }^{13}$, A. Reimer $^{13}$,

O. Reimer ${ }^{13}$, M. Renaud ${ }^{17, \star}$, R. de los Reyes ${ }^{3}$, F. Rieger ${ }^{3,41}$, L. Rinchiuso ${ }^{18}$, C. Romoli ${ }^{4}$, G. Rowell ${ }^{14}$, B. Rudak ${ }^{34}$, C.B. Rulten ${ }^{15}$, S. Safi-Harb ${ }^{44}$,

V. Sahakian ${ }^{6,5}$, S. Saito ${ }^{42}$, D. A. Sanchez ${ }^{24}$, A. Santangelo ${ }^{29}$, M. Sasaki ${ }^{36}$, R. Schlickeiser $^{11}$, F. Schüssler ${ }^{18}$, A. Schulz $^{37}$, U. Schwanke $^{7}$,

S. Schwemmer ${ }^{25}$, M. Seglar-Arroyo ${ }^{18}$, M. Settimo ${ }^{16}$, A. S. Seyffert ${ }^{1}$, N. Shafi ${ }^{23}$, I. Shilon ${ }^{36}$, K. Shiningayamwe ${ }^{8}$, R. Simoni $^{9}$, H. Sol $^{15}$,

F. Spanier ${ }^{1}$, M. Spir-Jacob ${ }^{31}$, Ł. Stawarz $^{38}$, R. Steenkamp ${ }^{8}$, C. Stegmann ${ }^{35,37}$, C. Steppa ${ }^{35}$, I. Sushch ${ }^{1}$, T. Takahashi ${ }^{43}$, J.-P. Tavernet ${ }^{16}$,

T. Tavernier ${ }^{31}$, A. M. Taylor ${ }^{37}$, R. Terrier ${ }^{31}$, L. Tibaldo ${ }^{3}$, D. Tiziani ${ }^{36}$, M. Tluczykont ${ }^{2}$, C. Trichard ${ }^{20}$, M. Tsirou $^{17}$, N. Tsuji ${ }^{42}$, R. Tuffs ${ }^{3}$,

Y. Uchiyama ${ }^{42}$, D. J. van der Walt ${ }^{1}$, C. van Eldik ${ }^{36}$, C. van Rensburg ${ }^{1}$, B. van Soelen ${ }^{40}$, G. Vasileiadis ${ }^{17}$, J. Veh $^{36}$, C. Venter $^{1}$, A. Viana $^{3,46}$,

P. Vincent ${ }^{16}$, J. Vink ${ }^{9}$, F. Voisin ${ }^{14}$, H. J. Völk ${ }^{3}$, T. Vuillaume ${ }^{24}$, Z. Wadiasingh ${ }^{1}$, S.J. Wagner ${ }^{25}$, P. Wagner ${ }^{7}$, R. M. Wagner ${ }^{27}$, R. White ${ }^{3}$,

A. Wierzcholska ${ }^{21}$, P. Willmann ${ }^{36}$, A. Wörnlein ${ }^{36}$, D. Wouters ${ }^{18}$, R. Yang ${ }^{3}$, D. Zaborov ${ }^{30}$, M. Zacharias ${ }^{1}$, R. Zanin ${ }^{3}$, A. A. Zdziarski $^{34}$, A. Zech ${ }^{15}$, F. Zefi $^{30}$, A. Ziegler ${ }^{36}$, J. Zorn ${ }^{3}$, and N. Żywucka ${ }^{38}$

(Affiliations can be found after the references)

Received 18 October 2017 / Accepted 8 February 2018

\section{ABSTRACT}

\begin{abstract}
Shell-type supernova remnants (SNRs) are considered prime candidates for the acceleration of Galactic cosmic rays (CRs) up to the knee of the CR spectrum at $E \approx 3 \times 10^{15} \mathrm{eV}$. Our Milky Way galaxy hosts more than 350 SNRs discovered at radio wavelengths and at high energies, of which 220 fall into the H.E.S.S. Galactic Plane Survey (HGPS) region. Of those, only 50 SNRs are coincident with a H.E.S.S source and in 8 cases the very high-energy (VHE) emission is firmly identified as an SNR. The H.E.S.S. GPS provides us with a legacy for SNR population study in VHE $\gamma$-rays and we use this rich data set to extract VHE flux upper limits from all undetected SNRs. Overall, the derived flux upper limits are not in contradiction with the canonical CR paradigm. Assuming this paradigm holds true, we can constrain typical ambient density values around shell-type SNRs to $n \leq 7 \mathrm{~cm}^{-3}$ and electron-to-proton energy fractions above $10 \mathrm{TeV}$ to $\epsilon_{\mathrm{ep}} \leq 5 \times 10^{-3}$. Furthermore, comparisons of VHE with radio luminosities in non-interacting SNRs reveal a behaviour that is in agreement with the theory of magnetic field amplification at shell-type SNRs.
\end{abstract}

Key words. gamma rays: general - ISM: supernova remnants

\section{Introduction}

Supernova remnants (SNRs) are considered the most promising candidates for the origin of Galactic cosmic rays (CRs), a long-standing open problem in astroparticle physics. These objects are also a very prominent source class in high-energy astrophysics, emitting non-thermal radiation in the form of radio waves, X-rays, and $\gamma$-rays. According to diffusive shock

${ }^{\star}$ Corresponding authors: H.E.S.S. Collaboration, e-mail: contact.hess@hess-experiment.eu acceleration (DSA) theory, with magnetic field amplification (see e.g. Malkov \& Drury 2001) hadronic CR particles such as protons and heavier nuclei can be accelerated up to $\mathrm{PeV}$ energies (the "knee" in the CR spectrum) at the expanding SNR shock front or shell. When these relativistic particles collide with other nuclei, for example in the nearby interstellar medium (ISM), they emit $\gamma$-rays in the very high-energy (VHE; $0.1 \lesssim E_{\gamma} \lesssim 100$ $\mathrm{TeV}$ ) band. Thus, observations with Cherenkov telescopes, sensitive to VHE $\gamma$-rays, provide a promising avenue to investigate not only the astrophysics of energetic SNRs themselves but also their putative connection to the origin of Galactic CRs (see 
e.g. Drury et al. 1994). In particular, certain aspects of DSA theory, such as the efficiency of particle acceleration mechanisms, can be constrained through such observations.

The High Energy Stereoscopic System (H.E.S.S.) is an array of five imaging atmospheric Cherenkov telescopes (IACTs) situated in Namibia. The telescope array has a field of view of approximately $5^{\circ}$ and can detect $\gamma$-rays above an energy threshold of $\sim 50 \mathrm{GeV}$. This array has an energy resolution of $\sim 15 \%$ and a angular resolution of $\sim 0.1^{\circ 1}$ (Aharonian et al. 2006). The H.E.S.S. Galactic Plane Survey (HGPS; H.E.S.S. Collaboration 2018 b) programme has led to the detection of 78 sources of VHE $\gamma$-rays, of which 8 have been firmly identified as emission from SNRs, typically by resolving shell-like morphologies matching those observed at lower energies. These are RX J1713.7-3946 (H.E.S.S. Collaboration 2018b), RX J0852.0-4622 (H.E.S.S. Collaboration 2018c), HESS J1731-347 (Abramowski et al. 2011), RCW 86 (H.E.S.S. Collaboration 2018d), W28 (Aharonian et al. 2008b), G349.7+0.2 (Abramowski et al. 2015), W49B (H.E.S.S. Collaboration 2018b), and HESS J1534-571 (H.E.S.S. Collaboration 2018b). One SNR outside of the HGPS region has also been firmly identified, SN 1006 (Acero et al. 2010), bringing the tally to 9 . In addition, H.E.S.S. has detected emission from 8 composite SNRs. For these latter types, it is currently difficult to determine whether the $\gamma$-rays originate in the interior pulsar wind nebula (PWN), the surrounding shell, or a combination of these two. In addition to the firm identifications, 16 additional HGPS sources have also been associated with SNRs based on spatial coincidence, see H.E.S.S. Collaboration (2018b).

Using a 10-year HGPS data set collected between 2004 and 2013, we investigate the sample of known radio and X-ray SNRs that so far have not been detected by IACTs. To that end, we select a subset of SNRs devoid of any unrelated VHE emission (the VHE-dark sample, Sect. 2) and derive flux upper limits (Sect. 4), which we use to test the standard paradigm of the SNRs as the origin of Galactic CRs. Assuming hadronic emission, we calculate a constraint on the fraction of the SNR explosion energy that is converted to CR protons in Sect. 5.3. We also apply a simple parametric estimate of the inverse-Compton (IC) emission to our results in order to probe the relevant parameter space in a mixed scenario where both leptonic and hadronic channels contribute to the source emission. Based on our formalism, we furthermore present expectations on the portion of this parameter space that will be accessible to the future Cherenkov Telescope Array (CTA; Actis et al. 2011) observatory. Finally, in Sect. 5.4, we compare the derived flux upper limits to the radio flux densities that have been observed for the investigated source sample and put these into context of the IACT detections.

\section{Candidate source selection and data sample}

We obtained the sample of source candidates from the SNRcat catalogue $^{2}$ (Ferrand \& Safi-Harb 2012), which provides an upto-date catalogue of SNRs detected from radio to VHE. The catalogue comprises SNRs of different morphological types: shell-type, composite, and filled-centre. In the context of this work, we treated only the shell-type and composite SNRs, as filled-centre SNRs correspond to pulsar wind nebulae, which are discussed in H.E.S.S. Collaboration (2018b). Also, we ignored sources of uncertain morphology (type "?” in SNRcat).

\footnotetext{
1 The mean point spread function $68 \%$ containment radius is $0.08^{\circ}$, see H.E.S.S. Collaboration (2018b).

2 http://www.physics.umanitoba.ca/snr/SNRcat/

Version used here as of 12.14.2015.
}

For our study we used the HGPS data set, which consists of $\sim 2700 \mathrm{~h}$ of observations and features a sensitivity of better than $\sim 1.5 \%$ of the Crab flux in the innermost Galactic regions.

Of the more than 300 shell-type and composite SNRs listed in SNRcat, 220 fall within the HGPS region (Galactic longitude from $65^{\circ}$ to $250^{\circ}$, latitude from $-3.5^{\circ}$ to $3.5^{\circ}$ ). However, as this region is populated by almost 80 sources detected by H.E.S.S. (see H.E.S.S. Collaboration 2018b) signal contamination of the analysis regions of the investigated SNRs is an important consideration. Any unrelated VHE emission in the analysis regions weaken the derived flux upper limits and complicate physical interpretation.

In order to obtain a sample of SNRs that does not suffer from this problem, we selected a VHE-dark sample from SNRcat consisting of sources that fall outside of regions of VHE emission. We note that by focussing on SNRs that are not detected at VHE we are biasing ourselves towards low VHE-flux objects. Assessing the impact of this bias and a possible correction would require population modelling and go beyond the main scope of this paper, which is to derive H.E.S.S. flux upper limits for VHE-undetected SNRs. The selection method is as follows: We used the HGPS significance map (see H.E.S.S. Collaboration 2018b) in an iterative way to identify VHE-bright regions around HGPS catalogue sources in the Galactic plane. More precisely, we chose the significance map with a $0.2^{\circ}$ correlation radius, since this radius roughly corresponds to the maximum of the source radius distribution for shell-type and composite SNRs listed in SNRcat. As starting points for this iteration, we used all bins ${ }^{3}$ of the significance map that both fall into the $80 \%$ signal containment radii of the HGPS sources ("R80" in the HGPS catalogue) and have significance values $\geq 4 \sigma$. We then saved their respective neighbouring bins with significances $\geq 4 \sigma$ and used these as starting points for the next iteration step. The iteration stops when there are no neighbouring bins with significances $\geq 4 \sigma$ around the starting points of a given step. This procedure results in sets of bins that define contiguous and VHE-bright regions, in the following referred to as de-selection regions. By construction, these regions overlap with the circular HGPS source regions, but are in most cases asymmetric in shape (see Fig. 1). Such regions are similar to the pre-defined HGPS exclusion regions (H.E.S.S. Collaboration 2018b) but smaller in extent to allow for a less conservative compromise between SNR sample size and signal leakage.

We tested the analysis region (see below) of any candidate object from SNRcat for overlap with one of the de-selection regions. If there was at least one bin in the analysis region belonging to such a region, we discarded the respective object from the VHE-dark SNR sample. In Fig. 1, we illustrate the method. This procedure results in a sample of 108 SNRs with H.E.S.S. observations, of which 83 are of shell-type and 25 are of composite morphology. The latter group includes eight sources with thermal and 15 sources with plerionic characteristics, and two objects that feature both characteristics.

\section{Analysis}

In this study, we selected only data of high quality using the criteria described in Aharonian et al. (2006) and the quality cut on atmospheric transparency conditions developed by Hahn et al. (2014). The observation live time of the analysed regions, corrected by the H.E.S.S. $\gamma$-ray acceptance, spans a range from $\sim 10 \mathrm{~min}$ to $\sim 80 \mathrm{~h}$ with a median value of $14.5 \mathrm{~h}$. The

\footnotetext{
Corresponding to a square of $0.02^{\circ} \times 0.02^{\circ}$ in Galactic coordinates.
} 
H.E.S.S. Collaboration: Population study of Galactic supernova remnants at very high $\gamma$-ray energies with H.E.S.S.

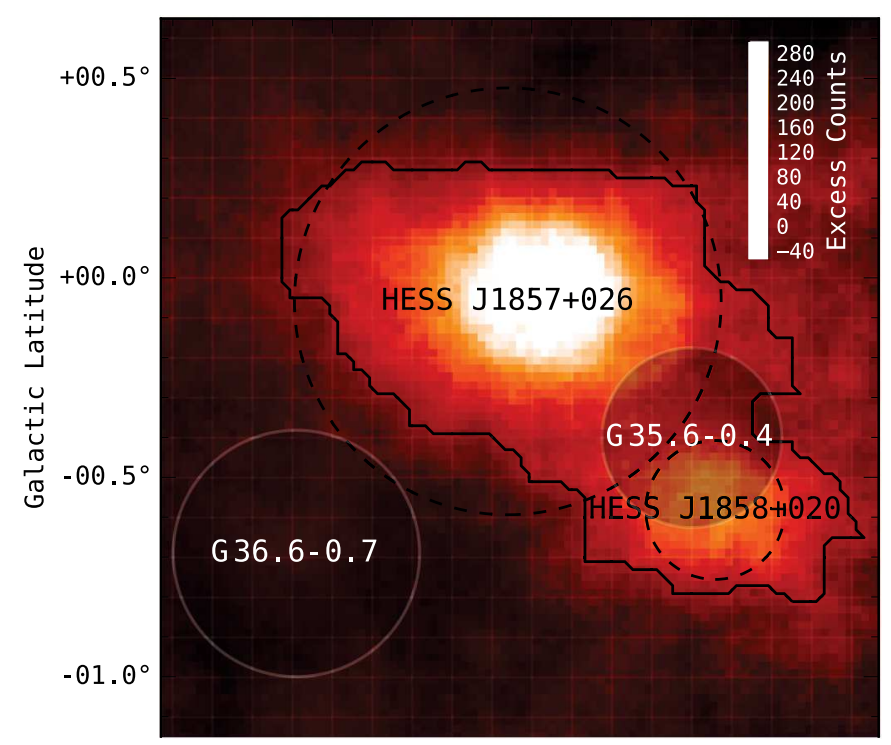

Fig. 1. Illustration of the source selection scheme on the $\gamma$-ray excess image from a given region. The known H.E.S.S. sources HESS J1857+026 and HESS J1858+020 with their $80 \%$ flux containment radii are shown with a dashed circle. The de-selection region resulting from the algorithm explained in Sect. 2 is shown with a solid curve. Because of the overlap with the de-selection region, the SNR G35.6-0.4 is discarded whereas SNR G36.6-0.7 is selected.

majority of data $(\sim 80 \%)$ have been recorded at average zenith angles smaller than $40^{\circ}$. Table A.1 lists the analysed source sample and the corrected observation live time, the averaged zenith angle for the observations of each source, and the closest H.E.S.S. source.

We analysed the data using the multivariate analysis method described in Ohm et al. (2009) with the same analysis configuration used in H.E.S.S. Collaboration (2018b), TMVA Hard Cuts. For the background estimation, the Reflected Background method (Berge et al. 2007), was applied. This method is largely insensitive to acceptance gradients in the cameras and therefore ideally suited for spectral analysis. A cross-check was performed using the Model++ Faint Cuts analysis (de Naurois \& Rolland 2009) and yielding compatible results.

The analysis region for every source is given by its position provided in SNRcat as well as the quoted radii therein and is defined to be circular. If an SNR is reported with an elliptical shape, we used the semi-major axis. A margin of $0.1^{\circ}$ is added to this radius, which conservatively takes the H.E.S.S. point spread function into account. Figure A.1 shows the analysis regions for our source sample.

For each source, we used all events above the safe energy threshold in the analysis. The safe energy is defined as the energy above which the energy bias is less than $10 \%$ (Aharonian et al. 2006).

We note that the diffuse emission measured in Abramowski et al. (2014a) was not taken into account in this analysis. This would result in conservative upper limits, especially for sources close to the Galactic plane. An attempt to quantify the effect of this component is described in Sect. 5 using the maps and largescale emission model from H.E.S.S. Collaboration (2018b).

\section{Results}

In Table A.1 we list the significance and upper-limit results for the individual sources. We calculate the significance using the

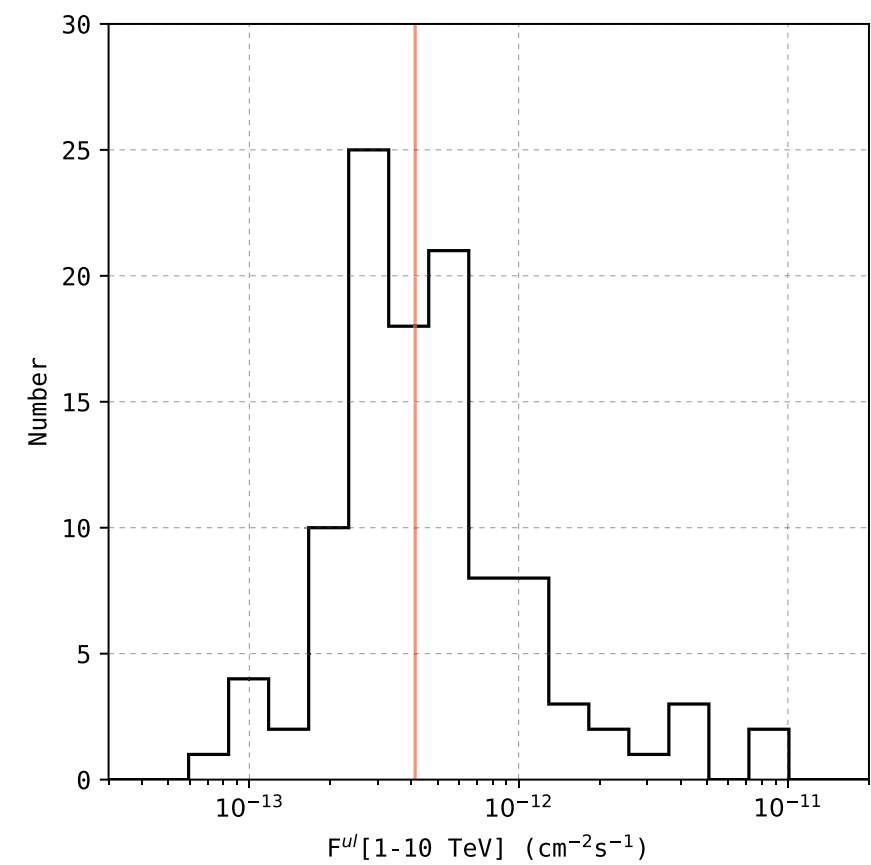

Fig. 2. Distribution of flux upper limits (99\% confidence level) of all investigated SNRs. The red line indicates the median value of $\sim 2 \%$ of the Crab nebula flux.

method proposed by Li \& Ma (1983). To obtain the upper limits on the excess counts above the safe energy, we use the profile likelihood method as described in Rolke et al. (2005) and assumed a confidence level of $99 \%$. We then express this result as an upper limit on the integrated flux in the $(1,10) \mathrm{TeV}$ interval assuming a power-law source of index 2.3. Such a value is typical for Galactic sources detected in the VHE range (H.E.S.S. Collaboration 2018b).

Figure 2 shows the distribution of upper limits for the VHEdark source sample. The total distribution is peaked at the typical H.E.S.S. sensitivity in the HGPS region of $\sim 2 \%$ of the Crab nebula flux. The median and variance values of the distribution of logarithmic flux upper limits are $M\left(\log _{10}\left(F^{\mathrm{ul}} /\left(\mathrm{cm}^{-2} \mathrm{~s}^{-1}\right)\right)=\right.$ -12.4 and $S^{2}\left(\log _{10}\left(F^{\mathrm{ul}} /\left(\mathrm{cm}^{-2} \mathrm{~s}^{-1}\right)\right)=0.14\right.$, respectively.

The significance distribution features median and variance values of $M(\sigma)=1.1$ and $S^{2}(\sigma)=2.4$, respectively. From Table A.1, one can notice that the $\gamma$-ray excess from the plerionic composite SNR G34.7-0.4 (W44) shows a significance of 6.2 $\sigma$. However, we are prevented from claiming a detection because this object is a rather large SNR embedded in a region of high diffuse emission that is not claimed as a source in the HGPS catalogue (see H.E.S.S. Collaboration 2018b) and because the signal is below the detection criteria of the independent cross-check analysis.

We show the significance distribution in the left panel of Fig. 3 together with a normal Gaussian, corresponding to the expectation in the absence of any source signal. There is no significant difference in the shape of the significance distribution with respect to the source type, as can be seen in the cumulative distributions shown in the right panel of Fig. 3. All pair-wise comparisons of the various significance distributions with twosided Kolmogorov-Smirnov tests result in $p$-values larger than 0.42 . Therefore we see no indication that the various samples stem from incompatible underlying distributions. In particular, we find no indication that the possible additional presence of a PWN in plerionic composites on average results in higher significance values in the VHE range. 

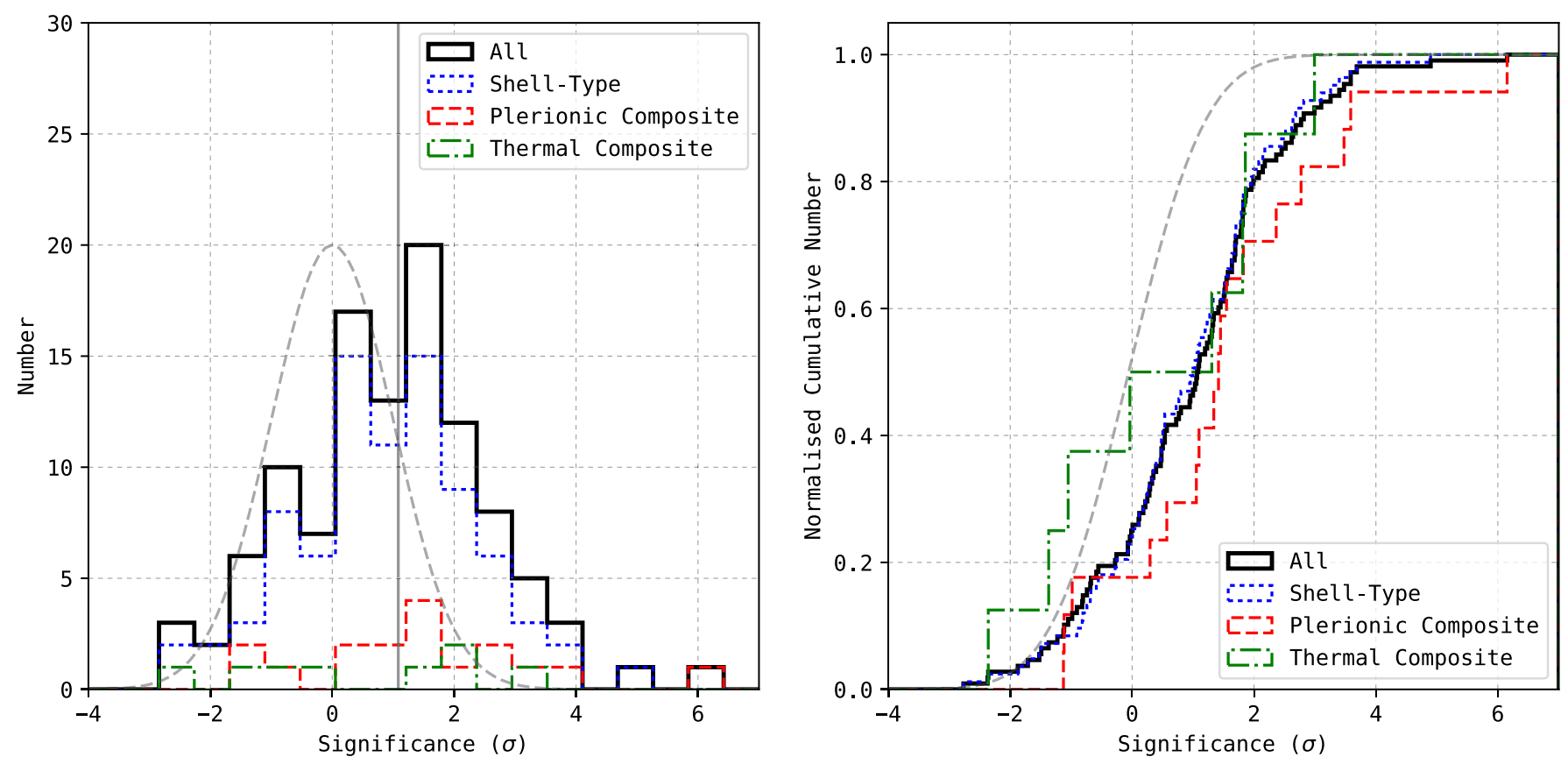

Fig. 3. Left: significance distribution of the VHE-dark sample of SNRs (black, blue, red, and green) and the corresponding median value (grey solid). The grey dashed curve indicates a normal Gaussian. Right: cumulative significance distribution of all sources (black), those of shell-type (blue), and composite (red and green) morphology. The grey dashed line represents a cumulative normal Gaussian.

\section{Discussion}

\subsection{Significance offset}

As shown in Fig. 3, the median value of the measured significance distribution is offset from the normal Gaussian distribution, which constitutes the expectation for pure noise (null hypothesis), and a Gaussian fit to the distribution results in best-fit values for mean and standard deviation of 1.01 and 1.51, respectively. A Kolmogorov-Smirnov test of the measured significance distribution against the null hypothesis rejects the latter with a $p$-value of $p=8 \times 10^{-15}$. The origin of this collective excess in positive significance values may arise from several contributions:

1. Low-level signal leakage into the analysis regions from known VHE $\gamma$-ray sources cannot be completely dismissed.

2. Imperfect background modelling can also lead to spurious excess emission, especially in background-dominated observations such as those made by H.E.S.S. The background reconstruction, however has been thoroughly studied in several publications (e.g. Berge et al. 2007 and Abramowski et al. 2012), typically resulting in small systematic uncertainties far below the excess observed here.

3. Galactic diffuse emission (Abramowski et al. 2014a) in the VHE range might cause the positive offset in the significance distribution. While the nature of this emission remains under discussion, it is believed that it consists both of a diffuse component of propagating CRs interacting with their environment and a population of unresolved sources (possibly including SNRs), which emit VHE $\gamma$-rays and a priori are unrelated to the individual SNRs investigated in this study.

4. The significance distribution offset might be the result of a cumulation of localised emission from the investigated SNR shells, which individually fall below the HGPS sensitivity.

The four components are hard to disentangle, and it is possible that we impose flux limits on a sum of these contributions. However, contribution (1) seems negligible, since no correlation

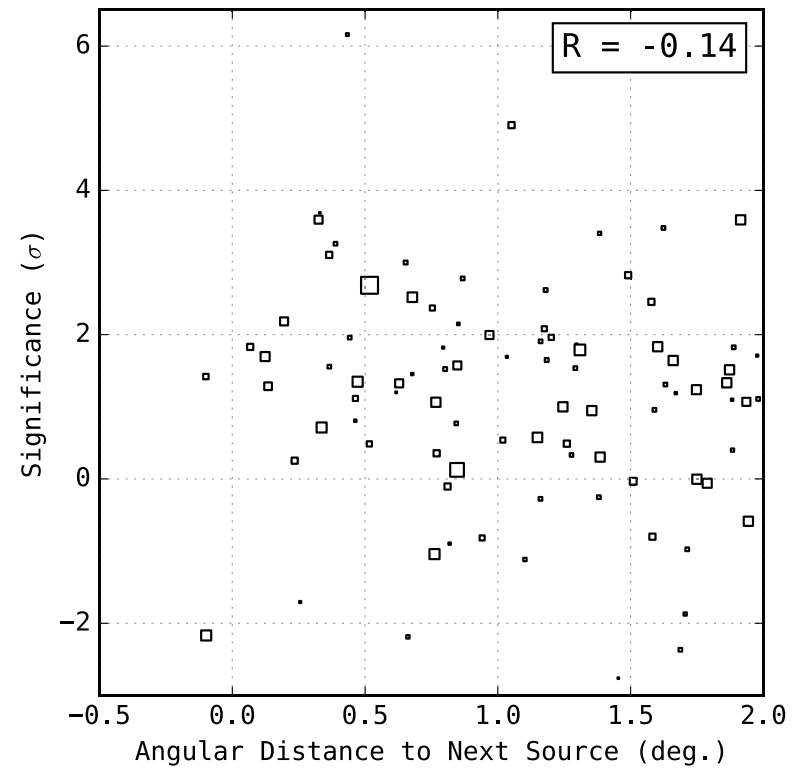

Fig. 4. Source significance values of the VHE-dark source sample vs. the angular distance to the nearest detected H.E.S.S. source. The angular distance between the edges of our test regions to that of detected H.E.S.S. sources is shown; the marker size is proportional to the integrated flux above $1 \mathrm{TeV}$ of the latter. The source region positions and sizes are taken from the HGPS, where they are defined as the centroids and $80 \%$ containment radii of symmetric and two-dimensional Gaussian fits to the integrated flux map, respectively (see H.E.S.S. Collaboration $2018 \mathrm{~b}$ for more details). Negative distance values are possible for asymmetric sources where the Gaussian fit does not describe the source morphology well, see e.g. SNR G36.6-0.7 in Fig. 1.

between excess significance and angular distance to the nearest HGPS source is apparent, as shown in Fig. 4.

The diffuse emission described in (3) extends out well beyond many regions of strong source emission and thus also the 
H.E.S.S. Collaboration: Population study of Galactic supernova remnants at very high $\gamma$-ray energies with H.E.S.S.

de-selection regions. As a result, this emission may be present in the investigated analysis regions. In Fig. 5, we show the cumulative significance distribution of the investigated SNR sample (compare to the right panel of Fig. 3) together with the analogous distribution that results if the diffuse emission contribution is accounted for in the significance calculation. To obtain the latter, we applied the parametric model of the diffuse emission component presented in H.E.S.S. Collaboration (2018b) to the data. It should be mentioned that for this calculation the significance values were derived directly from the HGPS maps. A Kolmogorov-Smirnov test of the distribution corrected in this way (red line in Fig. 5) against a normal (noise-) distribution (grey) results in a $p$-value of $p=1.8 \times 10^{-2}$, roughly corresponding to a significance value of $\sim 2.1 \sigma$, which suggests that the parametric diffuse emission model can account for a large portion, but not all of the significance offset.

In order to investigate contribution (4), we performed an analysis of randomised SNR analysis regions. That is, if the observed significance offset was indeed due to localised, faint emission from SNRs, we would expect this effect to be absent in a sample of randomised test regions. We determined the random positions by adding a uniform variate $l \in[-5,5)^{\circ}$ to the Galactic longitude value of each real SNR. We left the Galactic latitude value unchanged so as not to introduce a bias regarding component (3), which shows a Galactic latitude dependence; see H.E.S.S. Collaboration (2018b). Also, we added a variate number $s \in[-0.05,0.05)^{\circ}$ to the radius of the test region, with a lower ceiling of the resulting radius of $0.1^{\circ}$. Once the random test region is generated, we subjected it to our source selection method (see Sect. 2), and additionally tested whether it overlaps with a previously generated region, in which case we rejected it. If the random test region was rejected, a new test region was generated in the same manner and tested again until it passed selection. We created and analysed 28 randomised samples of the real set of SNRs, and each sample yields a set of 108 significance values. From these 28 sets, we calculated the average cumulated and normalised significance distribution. We then used this distribution as the cumulative density function in a Kolmogorov-Smirnov test against the significance distribution of the real SNR sample (blue line in Fig. 5). This results in a $p$-value of $p=5 \times 10^{-3}$, approximately corresponding to a significance of $\sigma \sim 2.6$, which does not allow us to claim the detection of a cumulated SNR signal.

Our results indicate that the observed shift in the significance distribution might be the result of the sum of components (3) and (4), although a deeper understanding of the large-scale VHE emission along the Galactic plane as well as improved analysis methods and observation exposure are required to provide definitive answers. Future studies with CTA should be able to shed light on this question.

\subsection{Fermi-LAT comparison}

The Fermi-LAT (Large Area Telescope) collaboration has recently performed a systematic survey in the $1-100 \mathrm{GeV}$ energy band towards known Galactic SNRs (Acero et al. 2016). Among the 102 source candidates, 30 are classified as likely GeV SNRs, and an additional 14 "marginally classified" candidates could be associated with the SNRs.

We compared the H.E.S.S. upper limits for all the GeV SNR candidates in our VHE-dark SNR sample with their extrapolated 1-10 TeV fluxes from the Fermi-LAT measurements. We note that the coordinates and radii of our analysis regions (see Sect. 3) differ from those found with Fermi-LAT. The H.E.S.S. upper

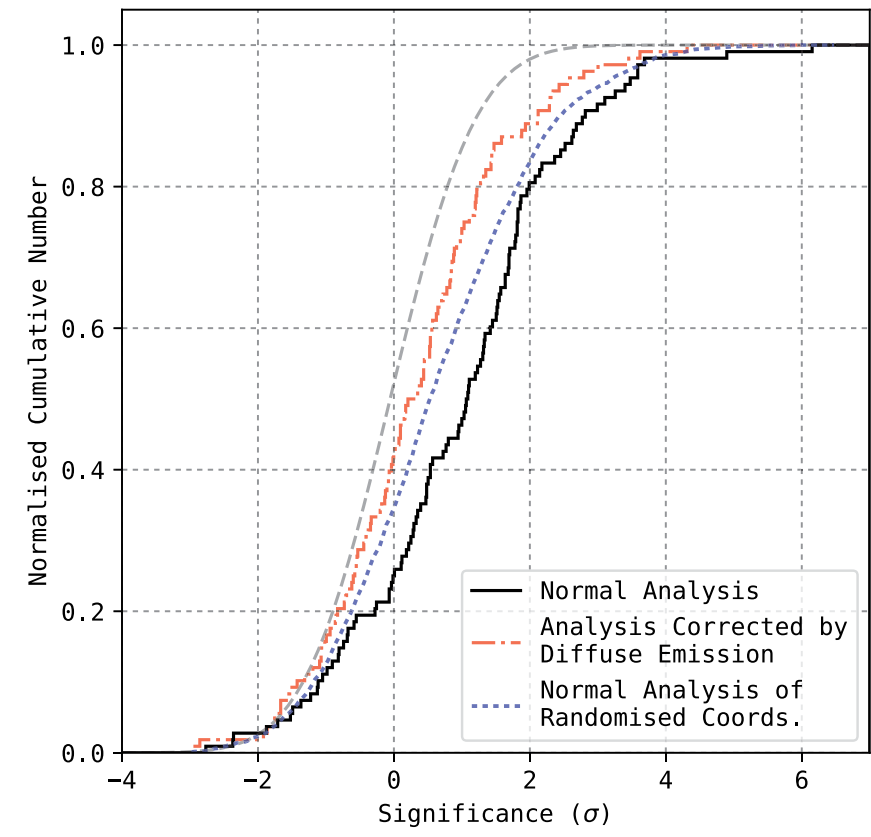

Fig. 5. Cumulated significance distribution of the SNR sample (black) and analogous distribution corrected for the Galactic diffuse emission component (dash-dotted red). The cumulated density function from the analysis of randomised test regions is shown in dotted blue. The grey dashed curve represents a cumulated normal Gaussian distribution.

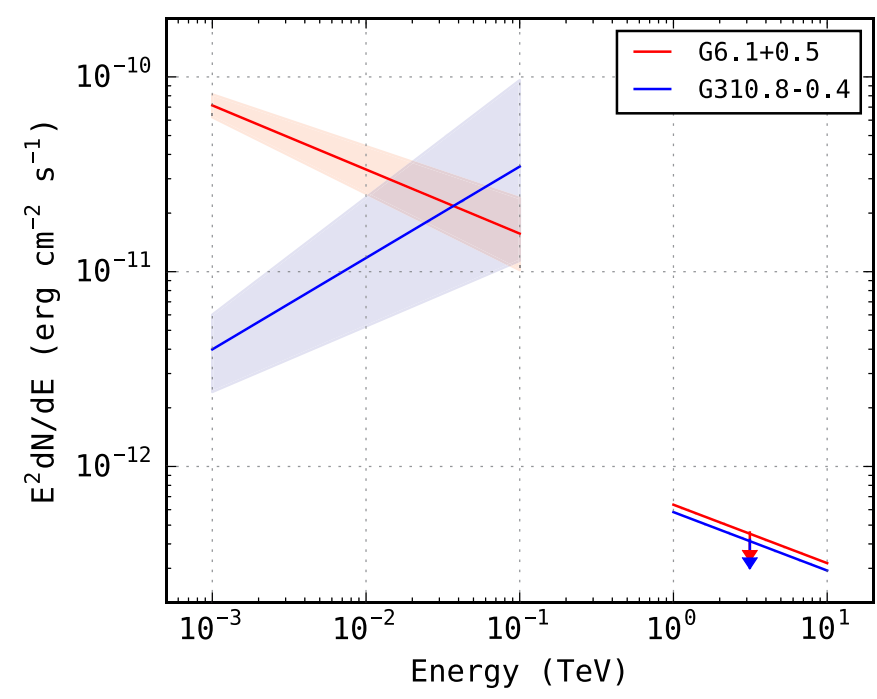

Fig. 6. Gamma-ray spectral energy distributions of G6.1+0.5 and G310.8-0.4. The Fermi-LAT spectra are derived from the spectral parameters reported in the first Fermi-LAT SNR catalogue. Statistical errors are indicated by the shaded bands.

limits are only constraining for two sources, namely G6.1+0.5 and G310.8-0.4; and hence, these upper limits point towards a spectral steepening in or before the VHE domain. However, these two sources are neither safely nor marginally classified GeV SNR candidates; the emission from G6.1+0.5 is flagged as doubtful and the GeV extent of G6.1+0.5 and G310.8-0.4 (0.64 and $\sim 1^{\circ}$, respectively) are much larger than their radio SNR size $\left(0.1^{\circ}-0.15^{\circ}\right.$ and $0.1^{\circ}$, respectively). The $\gamma$-ray spectra of these two sources are shown in Fig. 6. 


\subsection{Constraints on the accelerated particles in SNRS}

By comparing to model expectations, the derived integral flux upper limits may be used to estimate upper limits on the energy content in high-energy particles. In the following, we limit our considerations to inelastic proton-proton collisions and the subsequent $\pi^{0}$-decay and inverse-Compton (IC) emission since these processes are believed to be the most relevant $\gamma$-ray emission mechanism in SNRs for the VHE regime.

If the corresponding $\pi^{0}$ production timescale due to inelastic proton-proton scattering, $\tau_{\pi^{0}}$, and electron cooling time, $t_{\mathrm{IC}}$, are known ${ }^{4}$ and a distance estimate for a source is given, the presented flux upper limits can be used to place upper limits on the total energy in electrons and protons at the time of emission. In the following, we assume that $\gamma$-rays with energies larger than $1 \mathrm{TeV}$ mainly probe particles with energies above $10 \mathrm{TeV}$ in both the IC and $\pi^{0}$-decay emission channels.

We used $\tau_{\pi^{0}}=t_{\pi^{0}} / n$, where $n$ is the number density of ambient gas nuclei in units of $1 \mathrm{~cm}^{-3}$ and $t_{\pi^{0}} \approx 5 \times 10^{15} \mathrm{~s}$. Unlike in the hadronic case, the IC cooling time depends strongly on the electron energy and the energy densities of the ambient radiation fields. We adopted a conservative value of the cooling time by assuming the cosmic microwave background (CMB) as the only ambient photon field and using the value for $10 \mathrm{TeV}$ electrons scattering in the Thomson regime ${ }^{5}$. This results in a cooling time of $t_{\mathrm{IC}} \approx 10 \mathrm{TeV} / \dot{E}(10 \mathrm{TeV})=\frac{3}{4} m_{\mathrm{e}}^{2} /\left(\sigma_{\mathrm{T}} c \epsilon_{\mathrm{CMB}} 10 \mathrm{TeV}\right) \approx$ $4 \times 10^{12} \mathrm{~s}$, where $m_{\mathrm{e}}$ is the electron rest mass, $\sigma_{\mathrm{T}}$ is the Thomson cross section, $c$ is the speed of light, and $\epsilon_{\mathrm{CMB}}$ is the energy density of the CMB.

Using these values it is possible to convert the upper limits on the integrated energy flux above $1 \mathrm{TeV}$ into upper limits on the total energy in protons and electrons above $10 \mathrm{TeV}$ at the time of emission. In general, for power-law spectra with $\Gamma \neq 2$ upper limits on the energy flux, $F_{\mathrm{E}}^{\mathrm{ul}}$, between energies $E_{1}$ and $E_{2}$ are connected to the corresponding integral flux upper limits, $F^{\mathrm{ul}}$, between $E_{1}$ and $E_{2}$ via

$F_{\mathrm{E}}^{\mathrm{ul}}=\frac{\Gamma-1}{\Gamma-2} \times \frac{E_{1}^{2-\Gamma}-E_{2}^{2-\Gamma}}{E_{1}^{1-\Gamma}-E_{2}^{1-\Gamma}} \times F^{\mathrm{ul}}$,

where in our case $E_{1}=1 \mathrm{TeV}, E_{2}=10 \mathrm{TeV}$, and $\Gamma=2.3$.

Upper limits on the total energy content in electrons can be derived as

$W_{\mathrm{e}}^{\mathrm{ul}}[>10 \mathrm{TeV}] \approx F_{\mathrm{E}}^{\mathrm{ul}}[>1 \mathrm{TeV}] \times t_{\mathrm{IC}} \times 4 \pi d^{2}$.

The comparable procedure in the case of protons yields

$W_{\mathrm{p}}^{\mathrm{ul}}[>10 \mathrm{TeV}] \approx F_{\mathrm{E}}^{\mathrm{ul}}[>1 \mathrm{TeV}] \times \tau_{\pi^{0}} \times 4 \pi d^{2}$,

or more explicitly, if $n$ is unknown,

$\left(W_{\mathrm{p}}[>10 \mathrm{TeV}] \times n\right)^{\mathrm{ul}} \approx F_{\mathrm{E}}^{\mathrm{ul}}[>1 \mathrm{TeV}] \times t_{\pi^{0}} \times 4 \pi d^{2}$,

implying that $W_{\mathrm{p}}[>10 \mathrm{TeV}]$ and $n$ are degenerate in this case, such that with this method upper limits on the proton energy content can only be placed if the corresponding values of the target gas densities are available.

These limits are conservative estimations. The exact proportionality coefficients $c_{\mathrm{x}}$, defined by $W=c_{\mathrm{x}} \times F_{\mathrm{E}} \times t_{\mathrm{x}} \times 4 \pi d^{2}$

\footnotetext{
4 The timescales $\tau_{\pi^{0}}$ and $t_{\mathrm{IC}}$ are defined as the inverse of the $\pi^{0}$ production rate from $\mathrm{p}-\mathrm{p}$ interactions and the time after which electrons have lost half of their energy due to the IC process, respectively.

5 This approximation introduces a $\sim 14 \%$ error.
}

for $\pi^{0}$-decay are $c_{\pi^{0}}=[0.7,0.7,0.95,0.81]$, using the recent parametrisation of the total inelastic p-p cross section for Geant4, Pythia8, SIBYLL2.1, and QGSJET-I from Kafexhiu et al. (2014), respectively. For the IC mechanism we obtained $c_{\mathrm{IC}-\mathrm{CMB}}=0.73$ applying the full Klein-Nishina cross section and taking the shape of the electron spectrum into account.

In Table A.1 we list the individual values for $\left(W_{\mathrm{p}} \times n\right)^{\mathrm{ul}}$ and $W_{\mathrm{e}}^{\mathrm{ul}}$. The top panel in Fig. 7 shows our limits of $\left(W_{\mathrm{p}} \times n\right)^{\mathrm{ul}}$ versus the estimated source distance, where we also show the corresponding estimates for the VHE-detected sample of isolated shell-type SNRs (see Table A.3 for references). Using the published flux values in case of VHE detections or our calculated flux upper limits for the undetected sources, we calculate the values according to Eq. (3) using the distance estimates listed in Table A.2. We see that the H.E.S.S. upper limits are most constraining for relatively close sources. Assuming a hypothetical average ambient density around SNRs of $n \sim 1 \mathrm{~cm}^{-3}$ and an intrinsic proton power-law spectrum resulting from classical first-order Fermi acceleration with index $\Gamma=2$, a handful of sources within a few kpc distance constrain the CR paradigm. This paradigm identifies SNRs as the sources of Galactic CR assuming that $10 \%$ of the blast energy of $10^{51} \mathrm{erg}$ goes into the acceleration of $\mathrm{CR}$ up to $\mathrm{PeV}$ energies. However, ambient density values vary strongly from object to object, and in the bottom panel of Fig. 7 we use the literature estimates listed in Table A.2 to derive values for $W_{\mathrm{p}}$. The reported density values in this table are in some cases derived from fits to X-ray spectra rather than direct measurements (e.g. from $\mathrm{CO}$ or HI observations). These values are characterised by the density inside the $\mathrm{X}$-ray emitting bubble (i.e. the inter-clump density), which might not be the relevant quantity for hadronic high-energy processes. Multi-TeV protons suffer less from energy losses than electrons of similar energy and are therefore able to propagate further and consequently probe a different environment than X-ray emitting electrons of similar energy. For instance, recent work suggests a correlation between $\mathrm{TeV} \gamma$-rays brightness and cold $\mathrm{HI}$ gas density, for example in the cases of HESS J1731-347 (Fukuda et al. 2014) and RX J1713.7-3946 (Fukui et al. 2012, Sano et al. 2015). This correlation points to $\gamma$-ray production at dense clumps with number densities well above $10 \mathrm{~cm}^{-3}$ rather than the rarefied inter-clump medium. Therefore, we treat ambient density values that have been derived from X-ray spectra as lower limits, which results in conservative upper limits on the proton energy content. In the cases in which studies reveal a correlation between $\gamma$-ray emission and gas clumps, we calculate the proton energy content for the two scenarios assuming either the low inter-clump medium density or the high value inside the clumps, which are both listed in Table A.2.

As can be seen, five of our limits are constraining the canonical expectation in this case: G290.1-0.8 (source 9 in plot), G296.1-0.5 (10) as well as G53.6-2.2 (5), G306.3-0.9 (14), and G350.1-0.3 (20), if its distance is at the lower end of the uncertainty interval. The limits become less stringent if the proton spectra are softer. However, even if a typical Galactic particle spectral index of $\Gamma=2.3$ is assumed, inferred from the large number of HGPS sources, two sources still constrain the theoretical expectation: that is G306.3-0.9 (14), if the source is situated at the very low end of the distance error interval, and G290.1-0.8 (9). However, the latter is a thermal composite SNR (as is also G53.6-2.2), estimated to be of an evolved age (>10 kyr) and thus unlikely to be a place of efficient particle acceleration to $\mathrm{PeV}$ energies as assumed in the canonical picture (see e.g. Ptuskin \& Zirakashvili 2005). Therefore we consider this limit as not constraining. Also, the well-studied sources Cassiopeia A, 
H.E.S.S. Collaboration: Population study of Galactic supernova remnants at very high $\gamma$-ray energies with H.E.S.S.
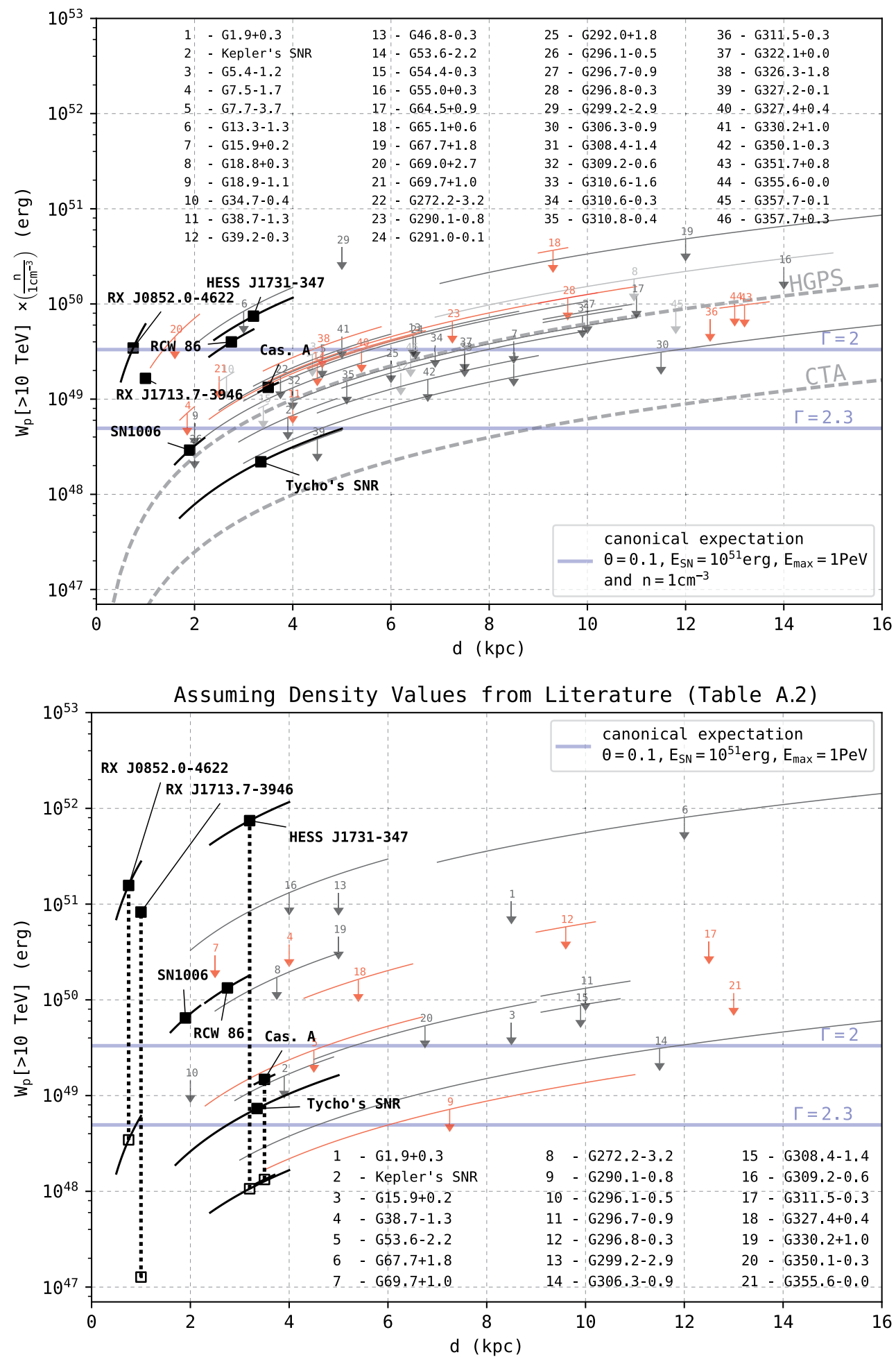

Fig. 7. Upper limits and measurements on the product of proton energy content above $10 \mathrm{TeV}$ and ambient density (top) and proton energy content above $10 \mathrm{TeV}$ (bottom) vs. source distance. We assume the ambient density and distance estimates from the publications listed in Table A.2 and also show the corresponding values for VHE-detected shell-type SNRs. For the latter, we use the flux normalisations as reported in the publications listed in Table A.3. In several sources a gas clump correlation of $\gamma$-ray emission was observed. In these cases, filled points indicate the result if the low density values in the rarefied inter-clump medium are used in the calculation, while open points show the result if the high density values inside the clumps are assumed. We also show the canonical expectation, assuming an acceleration efficiency of $\Theta=10 \%$, a SN blast energy of $E_{\mathrm{SN}}=10^{51}$ erg, and a power- law spectrum with spectral index of $\Gamma=2$ (expected from standard first order Fermi acceleration) and $\Gamma=2.3$ (corresponding to the average spectral index of HGPS sources) up to $E_{\max }=1 \mathrm{PeV}$, assuming $n=1 \mathrm{~cm}^{-3}$ in the top panel. Furthermore, we show in the top panel the sensitivity of the HGPS (assuming a mean value of $\sim 1.5 \% \mathrm{Crab}$ ) and the projected sensitivity of CTA (assuming a ten times higher sensitivity). Red points represent old sources ( $>10 \mathrm{kyr}$ ) that are not expected to accelerate protons to PeV energies, grey limits in the top panel indicate SNRs that are likely to interact with molecular clouds (see Table A.1). Values for Kepler's SNR (source 2), G1.9+0.3 (1) and G330.2+1.0 (41 top, 19 bottom) are derived from the limits in Aharonian et al. (2008a) and Abramowski et al. (2014b). 


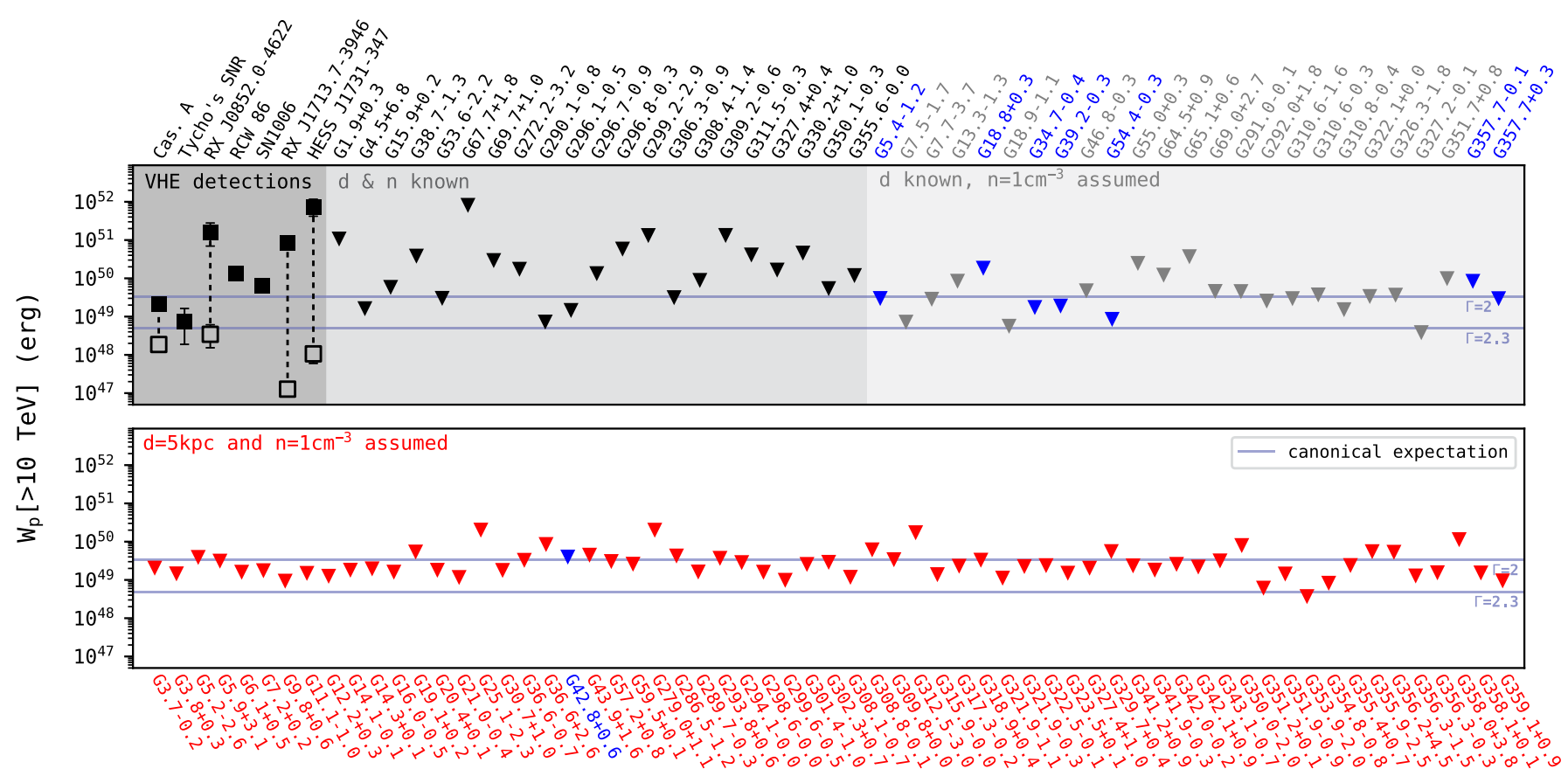

Fig. 8. Sample of upper limits on the proton energy content above $10 \mathrm{TeV}$ (triangles). Values are derived using the centre of the distance uncertainty interval. Blue triangles indicate SNRs that are likely to interact with molecular clouds (see Table A.1). Points are shown for the VHE-detected sample of SNRs, where the error arising from the distance uncertainty is indicated. In several sources a gas clump correlation of $\gamma$-ray emission was observed. In these cases, filled points indicate the result if the low density values in the rarefied inter-clump medium are used in the calculation, while open points show the result if the high density values inside the clumps are assumed.

Kepler's SNR, and Tycho lie below the canonical estimate if $\Gamma=2$. However, the latter two sources require larger indices in order to model their VHE emission (see Acciari et al. 2011 and Ahnen et al. 2017), and thus in these cases the comparison to the canonical value is of limited validity.

In another form of presentation, Fig. 8 shows the upper limits on $W_{\mathrm{p}}[>10 \mathrm{TeV}]$ for all objects that have been investigated, assuming a canonical ISM value of $n=1 \mathrm{~cm}^{-3}$ as ambient density and a typical source distance of $d=5 \mathrm{kpc}$, where this information is not available. It also shows the corresponding values for the VHE-detected SNRs.

The sample shows very high limits, which by far exceed the canonical expectation. This indicates that in these cases the sensitivity for the direction of the corresponding sources is not sufficient to provide constraining flux upper limits.

In those cases where clump interaction has been observed we see excessive values for $W_{\mathrm{p}}$, especially RX J0852.0-4622, RX J1713.7-3946 and HESS J1731-347 if the rarefied interclump density values are assumed. Adopting a high density inside the clumps leads to very low values of $W_{\mathrm{p}}$ that lie below the canonical expectation. Most notably, the derived value for RX J1713.7-3946 is about 1.5 orders of magnitudes below the canonical expectation for $\Gamma=2.3$. This value seems very low as in a hadronic emission scenario a high value for the cut-off in the proton spectrum of $\sim 90 \mathrm{TeV}$ is required to model the H.E.S.S. spectrum (see H.E.S.S. Collaboration 2018a).

Perhaps the inclusion of $\gamma$-ray emission from electrons via the IC mechanism may be a solution to this problem. Combining both the hadronic and leptonic estimations and introducing the electron to proton energy fraction above $10 \mathrm{TeV}, \epsilon_{\mathrm{ep}}$, we can write

$W_{\mathrm{p}}^{\mathrm{ul}}[>10 \mathrm{TeV}]=\frac{F_{\mathrm{E}}^{\mathrm{ul}}[>1 \mathrm{TeV}] \times 4 \pi d^{2}}{n / t_{\pi^{0}}+\epsilon_{\mathrm{ep}} / t_{\mathrm{IC}}}$.
In the following we want to determine the portion of the $\left\{n, \epsilon_{\mathrm{ep}}\right\}$ parameter space that is excluded by the flux upper limits, assuming that the accelerated proton distributions in SNRs meet the canonical expectation mentioned in the earlier paragraphs and follow a power-law distribution with a spectral index of $\Gamma=2.3$.

Our upper limits are constraining a parameter set in the $\left\{n, \epsilon_{\mathrm{ep}}\right\}$-plane if $W_{\mathrm{p}}^{\mathrm{ul}}[>10 \mathrm{TeV}]<W_{\mathrm{p}}^{\mathrm{th}}[>10 \mathrm{TeV}]$, where $W_{\mathrm{p}}^{\mathrm{th}}[>10 \mathrm{TeV}]$ is the theoretically expected value from the canonical assumption. Each individual upper limit then results in a curve in this parameter space above which the corresponding source would have been detected by H.E.S.S. and is therefore excluding the corresponding portion of the parameter space for this object. The set of curves is shown in Fig. 9. This figure also shows the distribution of constraints on $\epsilon_{\text {ep }}$ and $n$ in the asymptotic leptonic- and hadronic-dominated scenario limits as one-dimensional histograms. The logarithmic medians of those distributions are $\log \left(\epsilon_{\mathrm{ep}}\right)$ and $\log \left(n / 1 \mathrm{~cm}^{-3}\right)$ are -2.28 and 0.83 , respectively. The variance in both cases is 0.26 .

In the canonical picture of particle acceleration in SNRs, these values constrain hadronic-dominated emission scenarios to ambient density values $n \lesssim 7 \mathrm{~cm}^{-3}$ and leptonic-dominated emission scenarios to $\epsilon_{\mathrm{ep}} \lesssim 0.5 \%$. The most stringent upper limit yields $n \lesssim 0.6 \mathrm{~cm}^{-3}$ and $\epsilon_{\mathrm{ep}} \lesssim 0.05 \%$.

For comparison, we also show in Fig. 9 the $\left\{n, \epsilon_{\mathrm{ep}}\right\}$-ntuples for the VHE-detected shell-type SNRs. We derive these values from the models in the publications listed in Table A.3. For a valid comparison with the constraints in the $\left\{n, \epsilon_{\mathrm{ep}}\right\}$ parameter space, we scale the parameters values by a factor $f=W_{\mathrm{p}, i} /\left(10^{50} \mathrm{erg}\right)$, where $W_{\mathrm{p}, i}$ is the total energy in CR protons that has been derived for the sources $i$ in the literature because in most cases the latter deviates from the canonical $10^{50} \mathrm{erg}$. In some publications the authors discuss both hadronicand leptonic-dominated scenarios, in which case we show the 
H.E.S.S. Collaboration: Population study of Galactic supernova remnants at very high $\gamma$-ray energies with H.E.S.S.

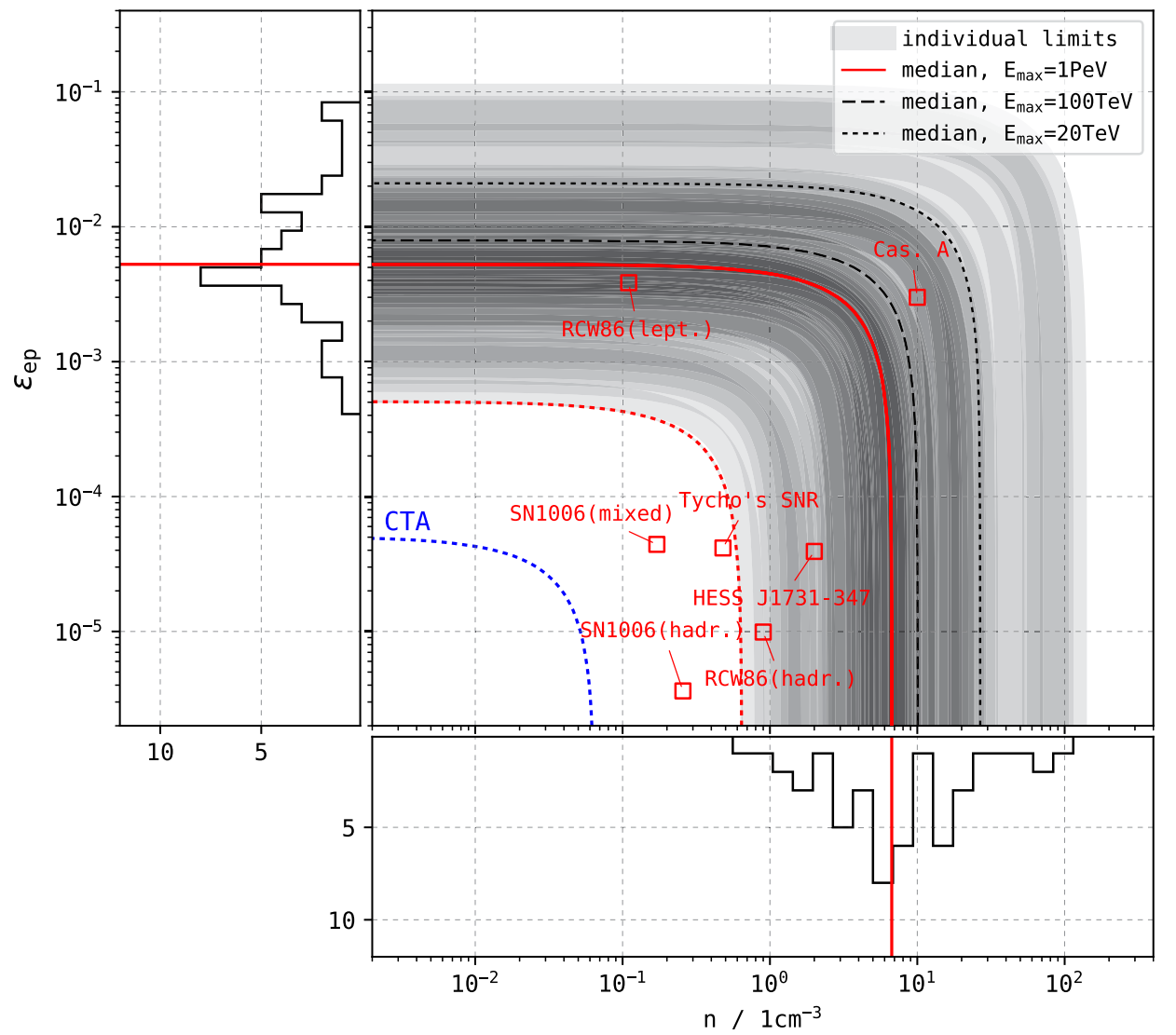

Fig. 9. Constraints on the $\epsilon_{\mathrm{ep}}$ and $n$ parameter space as imposed by the flux upper limits (grey bands, each limit constitutes one band). For each constraint a source-intrinsic power-law spectrum with index $\Gamma=2.3$ was assumed. Solid and dotted red lines represent the constraints corresponding to the median and lowest value of the flux upper limit distribution, respectively. The blue line indicates the boundary above which CTA might be able to probe the parameter space, assuming a ten times higher sensitivity than H.E.S.S. and taking the lowest flux upper limit as a reference. Additionally, values of $\epsilon_{\mathrm{ep}}$ and $n$ for VHE-detected shell-type SNRs are shown, extracted from the publications in Table A.3. For better comparison, these values are rescaled to the canonical total CR energy in SNRs of $10^{50} \mathrm{erg}$. The dashed and dotted black lines indicate how the median of the distribution shifts if one limits the maximum proton energy to $100 \mathrm{TeV}$ and $20 \mathrm{TeV}$, respectively. The histograms show the parameter limit distributions in the extreme hadronic- and leptonicdominated scenarios. respective points in the parameter space for both cases. The majority of the detected sources fall into the part of the parameter space that would allow for the detection by the HGPS. However, its sensitivity would not allow for the detection of the two VHE-faintest SNRs: Tycho's SNR, detected by the VERITAS collaboration (Acciari et al. 2011), and SN 1006. These sources required pointed observations to reach the exposure necessary for detection.

It should be stressed that the theoretical interpretation of the presented analysis results is rather simple and does not take into account the full complexity of the SNRs (which is beyond the scope of this paper). Also, many distance and density estimates used in this study suffer from large uncertainties with factors of a few; see Table A.2.

That said, if the assumptions made in our considerations are roughly plausible for the average young to middle-aged SNR, the next generation observatory, CTA, holds great potential for SNR science. An improvement in instrument sensitivity by an order of magnitude, as planned with CTA, will allow it to probe a considerably increased fraction of the parameter space, corresponding to the portion of the parameter space above the blue dotted line in Fig. 9. If our theoretical expectations are sound, we can expect CTA to test the SNR paradigm for ambient densities in the typical ISM range independent of the primary emission mechanism. If the $\gamma$-ray emission is dominated by the leptonic channel, even SNRs in rarefied environments such as the interior of bubbles blown by the main-sequence winds of the SNR progenitor stars should be detectable with CTA.

\subsection{Luminosity evolution of SNRs in the radio and VHE bands}

The average sensitivity of the HGPS is at the level of $2 \%$ of the Crab nebula VHE flux. There are sky regions of deeper exposure and thus lower values around prominent sources such as the Galactic centre. Because of the limited sensitivity, we can expect selection effects in the sample of detected SNRs roughly following the relations $L_{\mathrm{VHE}} / 4 \pi d^{2}>S$ and $L_{\mathrm{VHE}} / 4 \pi d>S$ for point-like and extended sources, respectively. In this work, $S$ is the H.E.S.S. point-like source sensitivity in a given field of view, $d$ is the distance to the source, and $L_{\mathrm{VHE}}$ is the source luminosity between $1 \mathrm{TeV}$ and $10 \mathrm{TeV}$. By including the sample of flux upper limits on radio SNRs in our considerations, we can achieve a more complete and less biased view on the VHE emission properties of this source class. Also, we want to make use of the large amount of radio information on SNRs and compare the VHE $\gamma$-ray fluxes to those observed at radio wavelengths.

To that end, we consider $L_{\mathrm{VHE}}$ and used the radio flux density values at $1 \mathrm{GHz}$, as provided in the Green SNR catalogue (Green 2014), to calculate the corresponding luminosity $L_{\text {Radio }}$. We furthermore formally assume a uniform bandwidth of $1 \mathrm{GHz}$ to convert from radio flux density to radio flux. The spectral assumptions used in the derivation of the VHE $\gamma$-ray luminosities of the VHE-detected shell-type SNRs are listed in Table A.3, along with the radio flux densities and age estimates from the SNRcat and Green catalogues. Finally, we remove SNRs from the sample for which interaction with molecular clouds is established or probable because in this study we want to investigate the physical processes at isolated SNR shocks. The information about whether a cloud interaction is occurring is also provided by SNRcat. However, such information is not available for all SNRs and thus it is possible that interacting SNRs are still present in the resulting source sample.

In the following, we investigate the data for linear correlations of both the VHE-luminosity $\left(y=\log \left(L_{\mathrm{VHE}}\right)\right)$ and the ratio of VHE-to-radio luminosities $\left(y=\log \left(L_{\mathrm{VHE}} / L_{\text {Radio }}\right)\right)$ with source age $(x=\log (\mathrm{Age} / 1 \mathrm{kyr}))$. The fit results can be found in Table 1. 

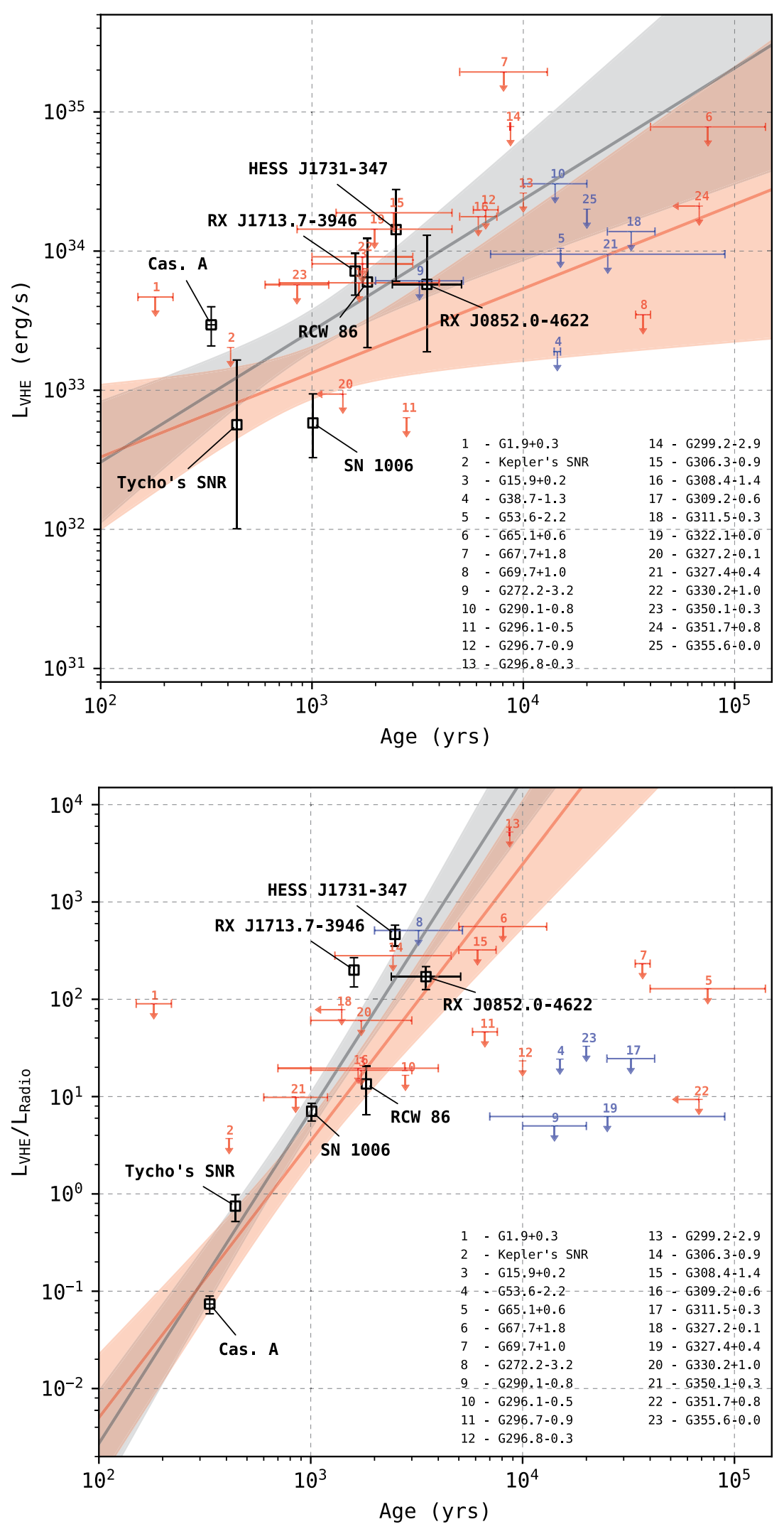

Fig. 10. Correlations of VHE luminosity between $1 \mathrm{TeV}$ and $10 \mathrm{TeV}$ of $L_{\mathrm{VHE}}($ top $)$ and $\left(L_{\mathrm{VHE}} / L_{\mathrm{radio}}\right)$ (bottom) with source age, where $L_{\text {radio }}$ is the luminosity at $1 \mathrm{GHz}$. Points: detected shell-type SNRs in the VHE regime. Arrows: upper limits. Red: shell-type SNRs. Blue: thermal composite SNRs. Values for Kepler's SNR, G1.9+0.3 and G330.2+1.0 from Aharonian et al. (2008a) and Abramowski et al. (2014b). Grey lines and uncertainty bands: Best-fit correlations taking only the detected SNRs into account. Red lines and uncertainty bands: Best-fit correlations when including also those upper limits into the fit where SNRs fall into the age interval defined by the sample of VHE-detections $(<5.1 \mathrm{kyr}$, upper age limit of RX J0852.0-4622).
In the top panel panel of Fig. 10 we show the VHE luminosities of shell-type and thermal-composite SNRs as a function of source age. A linear fit in estimated source age to the data points of those SNRs detected in both the radio and the VHE bands (black points) shows no sign of correlation (with a $p$-values of $p=0.1$ testing the null-hypothesis of a non-correlation). Also the inclusion of upper limits ${ }^{6}$ that fall into the same age interval as the detected SNRs $(<5.1 \mathrm{kyr})$ does

6 Correlation testing including upper limits was performed using the Cox hazard model and the EM algorithm regression provided by the asurv package (Lavalley et al. 1992), which is available at http:// ascl. net/1406.001 
H.E.S.S. Collaboration: Population study of Galactic supernova remnants at very high $\gamma$-ray energies with H.E.S.S.

Table 1. Fit results from correlation testing.

\begin{tabular}{cccc}
\hline \hline & $p$-value & Slope & Intercept \\
\hline$L_{\mathrm{VHE}}$ & 0.1 & $0.9 \pm 0.4$ & $33.4 \pm 0.2$ \\
+ limits & 0.1 & $0.6 \pm 0.5$ & $33.1 \pm 0.2$ \\
\hline$L_{\mathrm{VHE}} / L_{\text {Radio }}$ & $2.3 \times 10^{-3}$ & $3.4 \pm 0.5$ & $0.9 \pm 0.2$ \\
+ limits & $2.7 \times 10^{-2}$ & $2.8 \pm 0.6$ & $0.5 \pm 0.2$ \\
\hline
\end{tabular}

not change this situation. In this figure, to be conservative, we assumed the largest distances compatible with uncertainties.

There is a large scatter in the data points that partially stems from substantial uncertainties in the distance estimates. One way to address this problem is to look at the ratio $\left(L_{\mathrm{VHE}} / L_{\mathrm{radio}}\right)$, as it eliminates this uncertainty by construction. Indeed, the resulting values of the VHE detections show considerably less scatter in the ordinate values around the best-fit linear regression, as can be seen in the bottom panel of Fig. 10, where we show $\left(L_{\mathrm{VHE}} / L_{\mathrm{radio}}\right)$ versus source age. We find evidence for correlation for the sample of shell-type SNRs that have been detected in both the radio and VHE bands (black) with a $p$-value of $p=2.3 \times 10^{-3}$. Adding the corresponding upper limit values for SNRs that fall into the same age window as the VHE detections, i.e. with ages $<5.1 \mathrm{kyr}$, weakens the correlation with age $\left(p=2.7 \times 10^{-2}\right)$. An inclusion of the upper limit values for the older SNRs in the age fit results in a high probability of a non-correlation $(p=0.9)$. While it is expected that the spread in $\left(L_{\mathrm{VHE}} / L_{\text {radio }}\right)$ increases with source age as the diverse environment around SNRs becomes more and more important for the SNR evolution, the sample of upper limit values from evolved ( $\gtrsim 10^{4} \mathrm{yr}$ ) SNRs lies consistently below the extrapolated correlation by some orders of magnitude. It should be stressed that the correlations found might be different for interacting SNRs, since we excluded these systems from our test sample.

From our limited data set we find that the increase of VHE luminosity with source age is smaller than that of $\left(L_{\mathrm{VHE}} / L_{\text {radio }}\right)$, which implies that in the first several thousand years of SNR evolution the radio-synchrotron emission decreases more rapidly than the VHE emission increases. Therefore, although we prefer to look at the ratio $\left(L_{\mathrm{VHE}} / L_{\text {radio }}\right)$ instead of the individual radio and VHE luminosities to eliminate the large distance uncertainties, we note that the observed correlation is mainly driven by the strong decrease in $L_{\text {radio }}$ with time rather than the relatively constant behaviour of $L_{\mathrm{VHE}}$. A time decrease of SNR luminosities at lower energies has been directly observed in Cas A for nonthermal X-ray and radio emission (see Dent et al. 1974; Vinyaikin 2014; Patnaude et al. 2011 and Sato et al. 2017).

At higher ages, there are no more VHE detections of shelltype or thermal composite SNRs, while synchrotron emission at radio energies continues. The latter finding agrees with the theoretical expectation that effective particle acceleration to multi-TeV energies occurs mainly in young SNRs but not in more evolved systems. The observed behaviour of the younger sources can be interpreted by invoking the notion of magnetic field amplification at SNR shocks. In this theory, the amplified B-field is fuelled by a fraction of the shock-generated CR pressure (see e.g. Bell 2004 and Völk et al. 2005), and is therefore expected to decrease as the shock slows down with increasing source age, which in turn would lead to a decreased overall synchrotron luminosity.

\section{Conclusions}

In this work we investigated a sample of 108 Galactic SNRs, comprised of sources that have been detected in lower energy bands, for VHE $\gamma$-ray emission using the H.E.S.S. Phase I data coming from the HGPS programme (H.E.S.S. Collaboration $2018 b$ ). For the first time, upper limits on the integrated $\gamma$-ray flux between 1 and $10 \mathrm{TeV}$ are provided for such a large set of Galactic SNRs. We note that the presented upper limits may be useful for continuing studies, such as in Cristofari et al. (2013), where VHE data were compared to a SNR population synthesis model to investigate the CR standard paradigm.

We paid special attention to the selection of these sources to minimise a possible signal leakage from unrelated H.E.S.S. detections (see H.E.S.S. Collaboration 2018b) into the analysis regions. We found a positive offset of the significance distribution corresponding to a median value of $1 \sigma$. To at least a large degree, the origin of this offset can be attributed to the diffuse Galactic TeV emission detected by H.E.S.S. We applied generic models of the two VHE $\gamma$-ray emission processes believed to be dominant in SNRs to the data, i.e. the $\pi^{0}$-decay in a hadronic and IC emission in a leptonic emission scenario to place constraints on the acceleration efficiency in SNRs and the energy content in electrons and protons above $10 \mathrm{TeV}$. Assuming typical parameters of the ambient gas density and the SN blast energy, the resulting values do not contradict the standard expecation that $\sim 10 \%$ of the SN blast energy is converted to CR in SNRs. We also investigated the opposite problem assuming that this canonical paradigm is valid and put constraints on the parameter space spanned by the ambient gas density around the shock and electron-to-proton energy fraction.

Overall, the derived flux upper limits are not in contradiction with the canonical CR paradigm. Assuming this paradigm holds true, we can constrain typical ambient density values around shell-type SNRs to $n \leq 7 \mathrm{~cm}^{-3}$ and electron-to-proton energy fractions above $10 \mathrm{TeV}$ to $\epsilon_{\mathrm{ep}} \leq 5 \times 10^{-3}$.

Finally, we compared the presented flux upper limits to the flux measurements of the seven non-interacting shell-type SNRs detected both in the radio and VHE range. We found evidence of correlation between the ratio of VHE $\gamma$-ray luminosity to radio luminosity, $\left(L_{\mathrm{VHE}} / L_{\text {radio }}\right)$, and source age. This correlation can be explained by invoking the theory of magnetic field amplification at SNR shocks, which accounts for the rapid decrease in radio luminosity by predicting a declining magnetic field strength as the shock slows down with increasing source age.

Further development in the SNR population from the observational point of view should be achieved with the next generation instrument: the CTA observatory. In this work we have also estimated the performance of this future observatory to probe the ambient gas density and the SN blast energy parameter space. The results suggest that this instrument will be an important leap forward in the investigation of the Galactic SNRs and will likely be able to confirm or invalidate the CR paradigm.

Acknowledgements. The support of the Namibian authorities and of the University of Namibia in facilitating the construction and operation of H.E.S.S. is gratefully acknowledged, as is the support by the German Ministry for Education and Research (BMBF), the Max Planck Society, the German Research Foundation (DFG), the Alexander von Humboldt Foundation, the Deutsche Forschungsgemeinschaft, the French Ministry for Research, the CNRS-IN2P3 and the Astroparticle Interdisciplinary Programme of the CNRS, the U.K. Science and Technology Facilities Council (STFC), the IPNP of the Charles University, the Czech Science Foundation, the Polish National Science Centre, the South African Department of Science and Technology and National Research Foundation, the University of Namibia, the National Commission on Research, Science \& Technology of Namibia (NCRST), the Innsbruck University, the Austrian Science Fund (FWF), and the Austrian Federal Ministry for Science, Research and Economy, the University of Adelaide and the Australian Research Council, the Japan Society for the Promotion of Science, and the University of Amsterdam. We appreciate the excellent work of the technical support staff in Berlin, Durham, Hamburg, Heidelberg, Palaiseau, Paris, Saclay, and in 
Namibia in the construction and operation of the equipment. This work benefited from services provided by the H.E.S.S. Virtual Organisation, supported by the national resource providers of the EGI Federation.

\section{References}

Abramowski, A., Acero, F., Aharonian, F., et al. 2011, A\&A, 531, A81 Abramowski, A., Acero, F., Aharonian, F., et al. 2012, ApJ, 757, 158

Abramowski, A., Aharonian, F., Ait Benkhali, F., et al. 2014a, Phys. Rev. D, 90, 122007

Abramowski, A., Aharonian, F., Benkhali, F. A., et al. 2014b, MNRAS, 441, 790

Abramowski, A., Aharonian, F., Ait Benkhali, F., et al. 2015, A\&A, 574, A100

Acciari, V. A., Aliu, E., Arlen, T., et al. 2011, ApJ, 730, L20

Acero, F., Ballet, J., Decourchelle, A., et al. 2009, A\&A, 505, 157

Acero, F., Aharonian, F., Akhperjanian, A. G., et al. 2010, A\&A, 516, A62

Acero, F., Ackermann, M., Ajello, M., et al. 2016, ApJS, 224, 8

Actis, M., Agnetta, G., Aharonian, F., et al. 2011, Exp. Astron., 32, 193

Aharonian, F., Akhperjanian, A. G., Bazer-Bachi, A. R., et al. 2006, A\&A, 457, 899

Aharonian, F., Akhperjanian, A. G., Barres de Almeida, U., et al. 2008a, A\&A, 488,219

Aharonian, F., Akhperjanian, A. G., Bazer-Bachi, A. R., et al. 2008b, A\&A, 481, 401

Ahnen, M. L., Ansoldi, S., Antonelli, L. A., et al. 2017, MNRAS, 472, 2956

Allen, G. E., Chow, K., DeLaney, T., et al. 2015, ApJ, 798, 82

Auchettl, K., Slane, P., Castro, D., Foster, A. R., \& Smith, R. K. 2015, ApJ, 810 43

Bell, A. R. 2004, MNRAS, 353, 550

Berge, D., Funk, S., \& Hinton, J. 2007, A\&A, 466, 1219

Broersen, S., \& Vink, J. 2015, MNRAS, 446, 3885

Broersen, S., Chiotellis, A., Vink, J., \& Bamba, A. 2014, MNRAS, 441, 3040

Busser, J.-U., Egger, R., \& Aschenbach, B. 1996, A\&A, 310, L1

Carlton, A. K., Borkowski, K. J., Reynolds, S. P., et al. 2011, ApJ, 737, L22

Cassam-Chenaï, G., Decourchelle, A., Ballet, J., et al. 2004, A\&A, 427, 199

Castro, D., Slane, P. O., Gaensler, B. M., Hughes, J. P., \& Patnaude, D. J. 2011, ApJ, 734, 86

Chen, Y., Seward, F. D., Sun, M., \& Li, J.-t. 2008, ApJ, 676, 1040

Cristofari, P., Gabici, S., Casanova, S., Terrier, R., \& Parizot, E. 2013, MNRAS 434, 2748

de Naurois, M., \& Rolland, L. 2009, Astropart. Phys., 32, 231

DeLaney, T., \& Rudnick, L. 2003, ApJ, 589, 818

Dent, W. A., Aller, H. D., \& Olsen, E. T. 1974, ApJ, 188, L11

Drury, L. O., Aharonian, F. A., \& Voelk, H. J. 1994, A\&A, 287, 959

Ferrand, G., \& Safi-Harb, S. 2012, Adv. Space Res., 49, 1313

Fukuda, T., Yoshiike, S., Sano, H., et al. 2014, ApJ, 788, 94

Fukui, Y., Sano, H., Sato, J., et al. 2012, ApJ, 746, 82

Green, D. A. 2014, Bull. Astron. Soc. India, 42, 47

Hahn, J., de los Reyes, R., Bernlöhr, K., et al. 2014, Astropart. Phys., 54, 25

H.E.S.S. Collaboration (Abdalla, H., Abramowski, A., et al.) 2018a, A\&A, 612, A6 (H.E.S.S. SI)

H.E.S.S. Collaboration (Abdalla, H., Abramowski, A., et al.) 2018b, A\&A, 612, A1 (H.E.S.S. SI)

H.E.S.S. Collaboration (Abdalla, H., Abramowski, A., et al.) 2018c, A\&A, 612, A7 (H.E.S.S. SI)

H.E.S.S. Collaboration (Abramowski, A., Aharonian, F., et al.) 2018d, A\&A, 612, A4 (H.E.S.S. SI)

Huang, R. H. H., Wu, J. H. K., Hui, C. Y., et al. 2014, ApJ, 785, 118

Hui, C. Y., \& Becker, W. 2009, A\&A, 494, 1005

Hui, C. Y., Seo, K. A., Huang, R. H. H., et al. 2012, ApJ, 750, 7

Hwang, U., \& Laming, J. M. 2003, ApJ, 597, 362

Kafexhiu, E., Aharonian, F., Taylor, A. M., \& Vila, G. S. 2014, Phys. Rev. D, 90, 123014

Lavalley, M., Isobe, T., \& Feigelson, E. 1992, ASP Conf. Ser., 25, 245

Lee, J.-J., Park, S., Hughes, J. P., \& Slane, P. O. 2014, ApJ, 789, 7

Li, T.-P., \& Ma, Y.-Q. 1983, ApJ, 272, 317

Long, K. S., Blair, W. P., Matsui, Y., \& White, R. L. 1991, ApJ, 373, 567

Lovchinsky, I., Slane, P., Gaensler, B. M., et al. 2011, ApJ, 731, 70

Malkov, M. A., \& Drury, L. O. 2001, Rep. Prog. Phys., 64, 429

Minami, S., Ota, N., Yamauchi, S., \& Koyama, K. 2013, PASJ, 65, 99

Moriguchi, Y., Yamaguchi, N., Onishi, T., Mizuno, A., \& Fukui, Y. 2001, PASJ, 53,1025

Ohm, S., van Eldik, C., \& Egberts, K. 2009, Astropart. Phys., 31, 383

Pannuti, T. G., Rho, J., Heinke, C. O., \& Moffitt, W. P. 2014, AJ, 147, 55

Park, N., \& VERITAS Collaboration 2015, Proc. 34th Int. Cosmic Ray Conf. (ICRC2015), 769

Park, S., Kargaltsev, O., Pavlov, G. G., et al. 2009, ApJ, 695, 431
Patnaude, D. J., Vink, J., Laming, J. M., \& Fesen, R. A. 2011, ApJ, 729, L28

Prinz, T., \& Becker, W. 2012, A\&A, 544, A7

Prinz, T., \& Becker, W. 2013, A\&A, 550, A33

Ptuskin, V. S., \& Zirakashvili, V. N. 2005, A\&A, 429, 755

Rakowski, C. E., Hughes, J. P., \& Slane, P. 2001, ApJ, 548, 258

Rakowski, C. E., Badenes, C., Gaensler, B. M., et al. 2006, ApJ, 646, 982

Reynolds, S. P., Borkowski, K. J., Hwang, U., et al. 2006, ApJ, 652, L45

Reynolds, S. P., Borkowski, K. J., Green, D. A., et al. 2008, ApJ, 680, L41

Reynolds, M. T., Loi, S. T., Murphy, T., et al. 2013, ApJ, 766, 112

Rolke, W. A., López, A. M., \& Conrad, J. 2005, Nucl. Instr. Meth. Phys. Res. A, 551,493

Sánchez-Ayaso, E., Combi, J. A., Albacete Colombo, J. F., et al. 2012, Ap\&SS, 337, 573

Sánchez-Ayaso, E., Combi, J. A., Bocchino, F., et al. 2013, A\&A, 552, A52

Sano, H., Fukuda, T., Yoshiike, S., et al. 2015, ApJ, 799, 175

Sato, T., Maeda, Y., Bamba, A., et al. 2017, ApJ, 836, 225

Slane, P., Lee, S.-H., Ellison, D. C., et al. 2014, ApJ, 783, 33

Tian, W. W., Haverkorn, M., \& Zhang, H. Y. 2007, MNRAS, 378, 1283

Vink, J. 2008, ApJ, 689, 231

Vinyaikin, E. N. 2014, Astron. Rep., 58, 626

Völk, H. J., Berezhko, E. G., Ksenofontov, L. T., \& Rowell, G. P. 2002, A\&A, 396, 649

Völk, H. J., Berezhko, E. G., \& Ksenofontov, L. T. 2005, A\&A, 433, 229

Winkler, P. F., Williams, B. J., Reynolds, S. P., et al. 2014, ApJ, 781, 65

Yoshita, K., Miyata, E., \& Tsunemi, H. 2000, PASJ, 52, 867

Zoglauer, A., Reynolds, S. P., An, H., et al. 2015, ApJ, 798, 98

1 Centre for Space Research, North-West University, Potchefstroom 2520, South Africa

2 Universität Hamburg, Institut für Experimentalphysik, Luruper Chaussee 149, 22761 Hamburg, Germany

3 Max-Planck-Institut für Kernphysik, PO Box 103980, 69029 Heidelberg, Germany

4 Dublin Institute for Advanced Studies, 31 Fitzwilliam Place, Dublin 2, Ireland

5 National Academy of Sciences of the Republic of Armenia, Marshall Baghramian Avenue, 24, 0019 Yerevan, Republic of Armenia

6 Yerevan Physics Institute, 2 Alikhanian Brothers St., 375036 Yerevan, Armenia

7 Institut für Physik, Humboldt-Universität zu Berlin, Newtonstr. 15, 12489 Berlin, Germany

8 University of Namibia, Department of Physics, Private Bag 13301, Windhoek, Namibia

9 GRAPPA, Anton Pannekoek Institute for Astronomy, University of Amsterdam, Science Park 904, 1098 XH Amsterdam, The Netherlands

10 Department of Physics and Electrical Engineering, Linnaeus University, 35195 Växjö, Sweden

11 Institut für Theoretische Physik, Lehrstuhl IV: Weltraum und Astrophysik, Ruhr-Universität Bochum, 44780 Bochum, Germany

12 GRAPPA, Anton Pannekoek Institute for Astronomy and Institute of High-Energy Physics, University of Amsterdam, Science Park 904, 1098 XH Amsterdam, The Netherlands

13 Institut für Astro- und Teilchenphysik, Leopold-FranzensUniversität Innsbruck, 6020, Innsbruck, Austria

14 School of Physical Sciences, University of Adelaide, Adelaide 5005 , Australia

15 LUTH, Observatoire de Paris, PSL Research University, CNRS, Université Paris Diderot, 5 Place Jules Janssen, 92190 Meudon, France

16 Sorbonne Universités, UPMC Université Paris 06, Université Paris Diderot, Sorbonne Paris Cité, CNRS, Laboratoire de Physique Nucléaire et de Hautes Energies (LPNHE), 4 place Jussieu, 75252, Paris Cedex 5, France

17 Laboratoire Univers et Particules de Montpellier, Université Montpellier, CNRS/IN2P3, CC 72, Place Eugène Bataillon, 34095 Montpellier Cedex 5, France

18 IRFU, CEA, Université Paris-Saclay, 91191 Gif-sur-Yvette, France

19 Astronomical Observatory, The University of Warsaw, Al. Ujazdowskie 4, 00-478 Warsaw, Poland

20 Aix Marseille Université, CNRS/IN2P3, CPPM, Marseille, France 
H.E.S.S. Collaboration: Population study of Galactic supernova remnants at very high $\gamma$-ray energies with H.E.S.S.

${ }^{21}$ Instytut Fizyki Jạdrowej PAN, ul. Radzikowskiego 152, 31-342 Kraków, Poland

${ }^{22}$ Funded by EU FP7 Marie Curie, grant agreement No. PIEF-GA2012-332350

23 School of Physics, University of the Witwatersrand, 1 Jan Smuts Avenue, Braamfontein, Johannesburg 2050, South Africa

${ }^{24}$ Laboratoire d'Annecy-le-Vieux de Physique des Particules, Université Savoie Mont-Blanc, CNRS/IN2P3, 74941 Annecy-le-Vieux, France

25 Landessternwarte, Universität Heidelberg, Königstuhl, 69117 Heidelberg, Germany

26 Université Bordeaux, CNRS/IN2P3, Centre d'Études Nucléaires de Bordeaux Gradignan, 33175 Gradignan, France

27 Oskar Klein Centre, Department of Physics, Stockholm University, Albanova University Center, 10691 Stockholm, Sweden

${ }^{28}$ Wallenberg Academy Fellow

29 Institut für Astronomie und Astrophysik, Universität Tübingen, Sand 1, 72076 Tübingen, Germany

${ }^{30}$ Laboratoire Leprince-Ringuet, Ecole Polytechnique, CNRS/IN2P3, 91128 Palaiseau, France

31 APC, AstroParticule et Cosmologie, Université Paris Diderot, CNRS/IN2P3, CEA/Irfu, Observatoire de Paris, Sorbonne Paris Cité, 10, rue Alice Domon et Léonie Duquet, 75205 Paris Cedex 13, France

32 Univ. Grenoble Alpes, CNRS, IPAG, 38000 Grenoble, France

33 Department of Physics and Astronomy, The University of Leicester, University Road, Leicester LE1 7RH, UK

${ }^{34}$ Nicolaus Copernicus Astronomical Center, Polish Academy of Sciences, ul. Bartycka 18, 00-716 Warsaw, Poland

35 Institut für Physik und Astronomie, Universität Potsdam, KarlLiebknecht-Strasse 24/25, 14476 Potsdam, Germany
${ }^{36}$ Friedrich-Alexander-Universität Erlangen-Nürnberg, Erlangen Centre for Astroparticle Physics, Erwin-Rommel-Str. 1, 91058 Erlangen, Germany

37 DESY, 15738 Zeuthen, Germany

38 Obserwatorium Astronomiczne, Uniwersytet Jagielloński, ul. Orla 171, 30-244 Kraków, Poland

39 Centre for Astronomy, Faculty of Physics, Astronomy and Informatics, Nicolaus Copernicus University, Grudziadzka 5, 87-100 Torun, Poland

40 Department of Physics, University of the Free State, PO Box 339, Bloemfontein 9300, South Africa

${ }^{41}$ Heisenberg Fellow (DFG), ITA Universität Heidelberg, Germany

42 Department of Physics, Rikkyo University, 3-34-1 Nishi-Ikebukuro, Toshima-ku, Tokyo 171-8501, Japan

43 Japan Aerpspace Exploration Agency (JAXA), Institute of Space and Astronautical Science (ISAS), 3-1-1 Yoshinodai, Chuo-ku, Sagamihara, Kanagawa 229-8510, Japan

44 Department of Physics \& Astronomy, University of Manitoba, Winnipeg MB R3T 2N2, Canada

45 Now at The School of Physics, The University of New South Wales, Sydney, 2052, Australia

46 Now at Instituto de Física de São Carlos, Universidade de São Paulo, Av. Trabalhador São-carlense, 400 - CEP 13566-590, São Carlos, SP, Brazil

47 Now at Kavli Institute for Particle Astrophysics and Cosmology, Department of Physics and SLAC National Accelerator Laboratory, Stanford University, Stanford, CA 94305, USA 


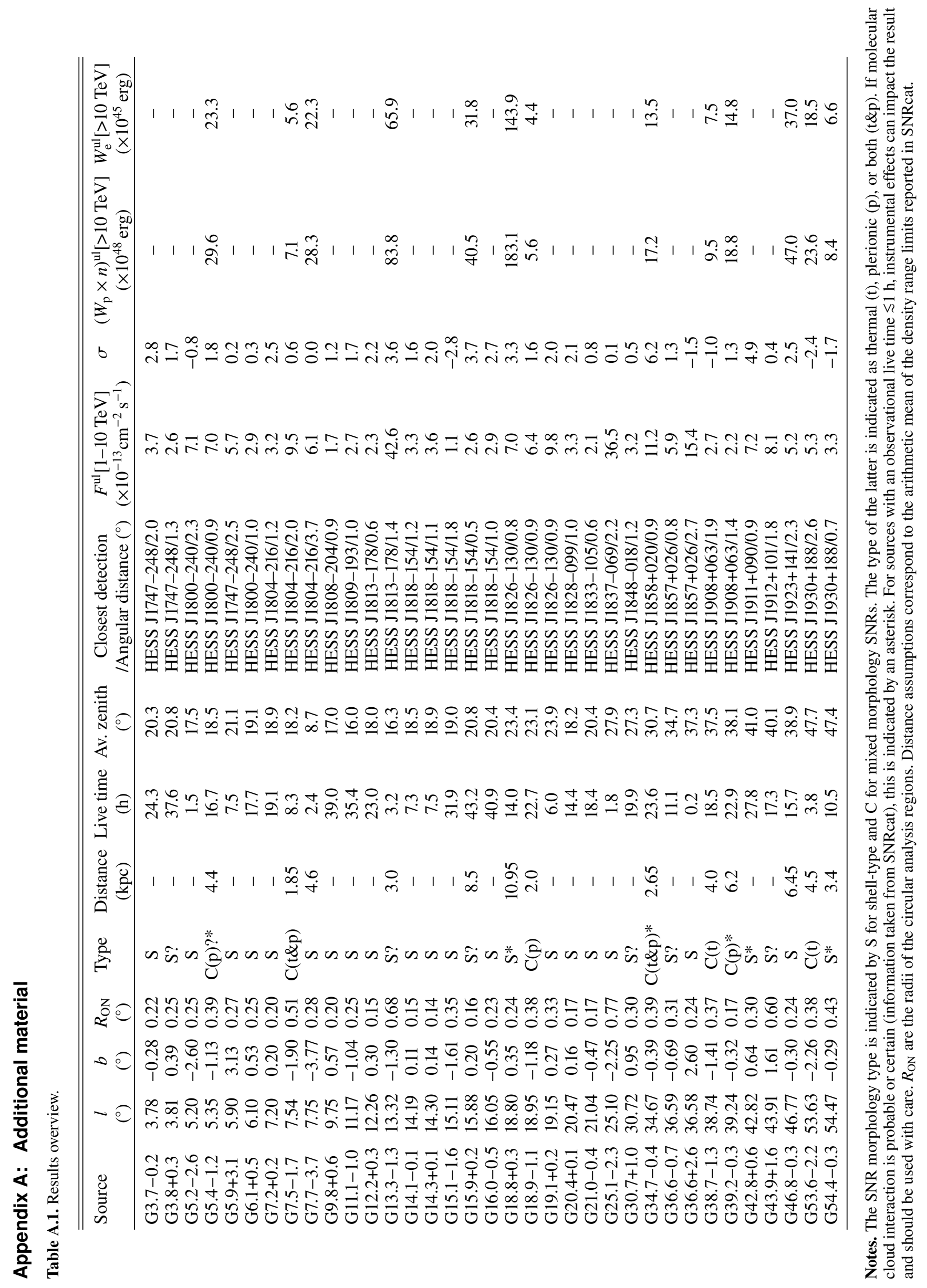

A3, page 14 of 18 
H.E.S.S. Collaboration: Population study of Galactic supernova remnants at very high $\gamma$-ray energies with H.E.S.S.

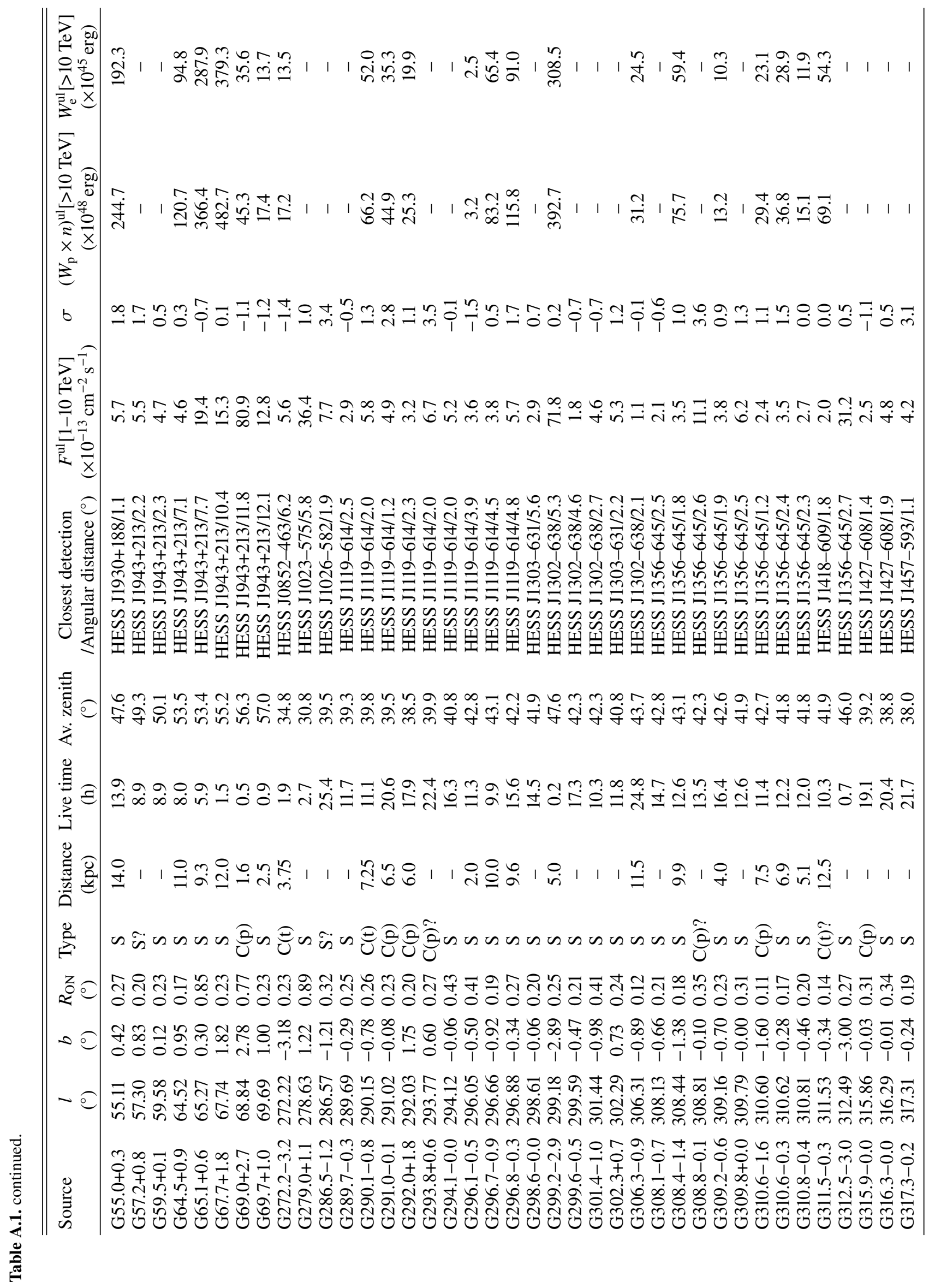

A3, page 15 of 18 


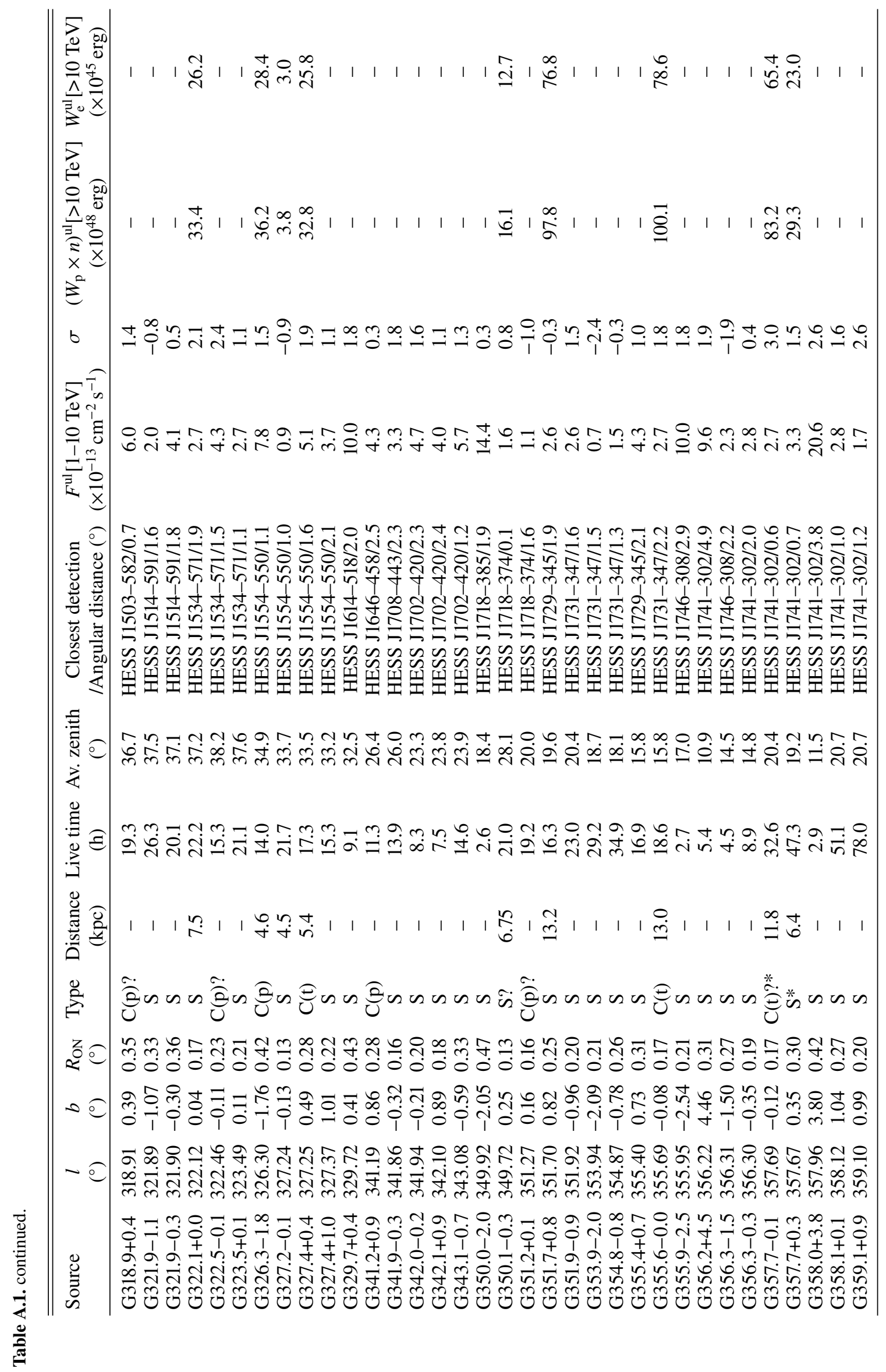


H.E.S.S. Collaboration: Population study of Galactic supernova remnants at very high $\gamma$-ray energies with H.E.S.S.

Table A.2. Literature estimates on source ages, distances, and ambient densities.

\begin{tabular}{|c|c|c|c|c|}
\hline Source & $\begin{array}{c}\text { Distance } \\
(\mathrm{kpc})\end{array}$ & $\begin{array}{l}\text { Age } \\
(\mathrm{kyr})\end{array}$ & $\begin{array}{l}\text { Density } \\
\left(\mathrm{cm}^{-3}\right)\end{array}$ & References \\
\hline Cassiopeia A & $3.3-3.7$ & $0.316-0.352$ & $0.6-1.2 / \sim 10 *$ & DeLaney \& Rudnick (2003), Lee et al. (2014), Hwang \& Laming (2003) \\
\hline Tycho's SNR & $1.7-5$ & 0.441 & 0.3 & Slane et al. (2014), Völk et al. (2002) \\
\hline RX J0852.0-4622 & $0.5-1$ & $2.4-5.1$ & $0.022 / \sim 10^{*}$ & Allen et al. (2015), Moriguchi et al. (2001) \\
\hline RCW 86 & $2.3-3.2$ & 1.83 & 0.3 & Broersen et al. (2014) \\
\hline SN 1006 & $1.6-2.2$ & 1.01 & 0.045 & Winkler et al. (2014) \\
\hline RX J1713.7-3946 & 1 & 1.6 & $<0.02 / \sim 130 *$ & Cassam-Chenaï et al. (2004), Acero et al. (2009), Fukui et al. (2012) \\
\hline H.E.S.S. J1731-347 & $2.4-4$ & 2.5 & $<0.01 / \sim 40-100 *$ & Abramowski et al. (2011), Fukuda et al. (2014) \\
\hline Kepler's SNR & $2.9-4.9$ & 0.412 & $0.4-5$ & Vink (2008) \\
\hline G1.9+0.3 & 8.5 & $0.15-0.22$ & $0.022-0.04$ & Reynolds et al. (2008), Carlton et al. (2011), Zoglauer et al. (2015) \\
\hline G15.9+0.2 & 8.5 & $1-3$ & 0.7 & Reynolds et al. (2006) \\
\hline G38.7-1.3 & 4 & $14-15$ & $0.025-0.034$ & Huang et al. (2014) \\
\hline G53.6-2.2 & $2.3-6.7$ & 15 & $0.79-1.36$ & Long et al. (1991), Broersen \& Vink (2015) \\
\hline G67.7+1.8 & $7-17$ & $5-13$ & $0.06-0.1$ & Hui \& Becker (2009) \\
\hline G69.7+1.0 & 2.5 & $34-40$ & 0.06 & Yoshita et al. (2000) \\
\hline G272.2-3.2 & $2.5-5$ & $2-5.2$ & 0.1 & Sánchez-Ayaso et al. (2013) \\
\hline G290.1-0.8 & $3.5-11$ & $10-20$ & 9.2 & Auchettl et al. (2015) \\
\hline G296.1-0.5 & 2 & 2.8 & 0.22 & Castro et al. (2011) \\
\hline G296.7-0.9 & $9.1-10.9$ & $5.8-7.6$ & $0.63-0.91$ & Prinz \& Becker (2013) \\
\hline G296.8-0.3 & 9 & 10 & 0.2 & Sánchez-Ayaso et al. (2012) \\
\hline G299.2-2.9 & 5 & 8.7 & 0.3 & Busser et al. (1996) \\
\hline G306.3-0.9 & 8 & $1.3-4.6$ & $1-49.1$ & Reynolds et al. (2013) \\
\hline G308.4-1.4 & $9.1-10.7$ & $5-7.5$ & $0.86-1.06$ & Hui et al. (2012), Prinz \& Becker (2012) \\
\hline G309.2-0.6 & $2-6$ & $0.7-4$ & $0.01-0.05$ & Rakowski et al. (2001) \\
\hline G311.5-0.3 & 12.5 & $25-42$ & 0.17 & Pannuti et al. (2014) \\
\hline $\mathrm{G} 327.4+0.4$ & $4.3-6.5$ & $7-90$ & $0.2-0.4$ & Chen et al. (2008) \\
\hline $\mathrm{G} 330.2+1.0$ & $>5$ & $1-3$ & 0.1 & Park et al. (2009) \\
\hline G337.2-0.7 & $2-9.3$ & $0.75-3.5$ & 0.6 & Rakowski et al. (2006) \\
\hline G350.1-0.3 & $4.5-9$ & $0.6-1.2$ & 0.3 & Lovchinsky et al. (2011) \\
\hline $\mathrm{G} 351.7+0.8$ & $12.7-13.7$ & $<68$ & $<0.4$ & Tian et al. (2007) \\
\hline G355.6-0.0 & 13 & 20 & 0.85 & Minami et al. (2013) \\
\hline
\end{tabular}

Notes. Some of the ambient density values are not direct measurements but are derived from fits to the X-ray spectra. In some cases correlations with high density gas clumps are observed or inferred. The corresponding densities inside the clumps are denoted by an asterisk.

Table A.3. Spectral parameters of the shell-type SNRs detected in both the radio and VHE bands.

\begin{tabular}{|c|c|c|c|c|}
\hline SNR & $\begin{array}{l}\text { Radio flux density } \\
\text { (@1 GHz, Jy) }\end{array}$ & $\begin{array}{l}\text { Integrated flux } F_{1-10 \mathrm{TeV}} \\
\quad\left(10^{-12} \mathrm{~cm}^{-2} \mathrm{~s}^{-1}\right)\end{array}$ & Flux references & Model references \\
\hline Cassiopeia A & 2720 & $0.58 \pm 0.12^{a}$ & Ahnen et al. (2017) & Ahnen et al. (2017) \\
\hline Tycho's SNR & 56 & $0.11 \pm 0.04$ & $\begin{array}{l}\text { Park \& VERITAS } \\
\text { Collaboration }(2015)\end{array}$ & Slane et al. (2014) \\
\hline RX J0852.0-4622 & 50 & $22.8 \pm 6.1$ & $\begin{array}{l}\text { H.E.S.S. Collaboration } \\
(2018 \mathrm{c})\end{array}$ & $\begin{array}{l}\text { H.E.S.S. Collaboration } \\
(2018 \mathrm{c})\end{array}$ \\
\hline RCW 86 & 49 & $1.82 \pm 0.94$ & $\begin{array}{l}\text { H.E.S.S. Collaboration } \\
(2018 d)\end{array}$ & $\begin{array}{l}\text { H.E.S.S. Collaboration } \\
(2018 d)\end{array}$ \\
\hline SN 1006 & 19 & $\sim 0.37 \pm 0.08^{b}$ & Acero et al. (2010) & Acero et al. (2010) \\
\hline RX J1713.7-3946 & $\sim 30^{c}$ & $16.4 \pm 5.4$ & $\begin{array}{l}\text { H.E.S.S. Collaboration } \\
\text { (2018a) }\end{array}$ & $\begin{array}{l}\text { H.E.S.S. Collaboration } \\
\text { (2018a) }\end{array}$ \\
\hline H.E.S.S. J1731-347 & 2.5 & $3.37 \pm 0.82$ & Abramowski et al. (2011) & Abramowski et al. (2011) \\
\hline
\end{tabular}

Notes. Except for the value for RX J1713-3946, all radio flux density values are taken from Green's catalogue Green (2014). Also given are the references to the models applied in Sect. 5. ${ }^{(a)}$ Assuming a systematic flux error of 20\%. ${ }^{(b)}$ Sum of north-east and south-west regions. ${ }^{(c)}$ Value from (Acero et al. 2009) extrapolated to $1 \mathrm{GHz}$. 

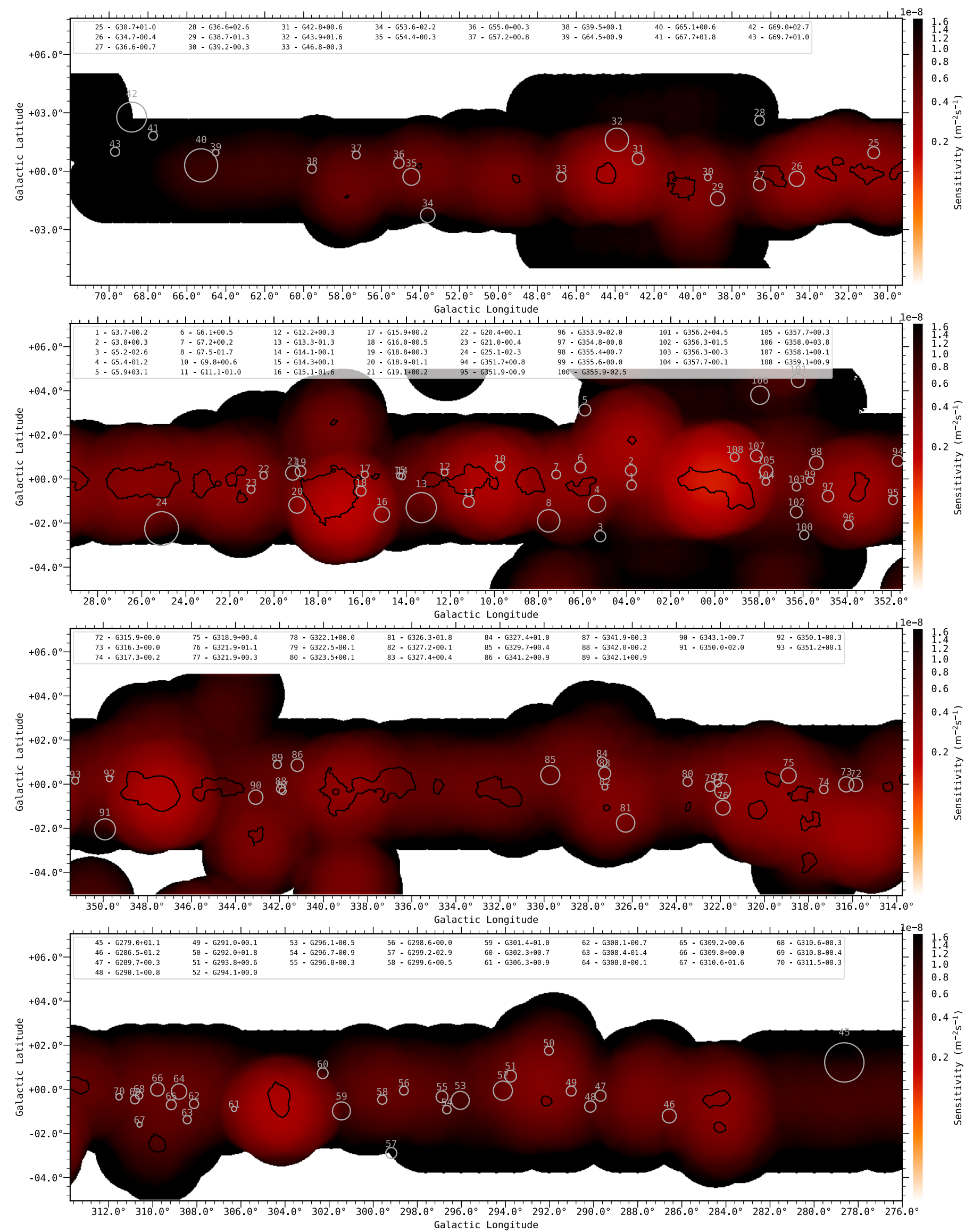

Fig. A.1. HGPS sensitivity map (for a correlation radius of $0.2^{\circ}$ ) overlaid with the analysis regions of our source sample (grey) and the de-selection regions (black); see also Sect. 2. 